\title{
Lin-Kernighan Heuristic Adaptations for the Generalized Traveling Salesman Problem
}

\author{
D. Karapetyan ${ }^{\mathrm{a}, *}$, G. Gutin ${ }^{\mathrm{a}}$ \\ ${ }^{a}$ Royal Holloway London University, Egham, Surrey, TW20 OEX, United Kingdom
}

\begin{abstract}
The Lin-Kernighan heuristic is known to be one of the most successful heuristics for the Traveling Salesman Problem (TSP). It has also proven its efficiency in application to some other problems.

In this paper we discuss possible adaptations of TSP heuristics for the Generalized Traveling Salesman Problem (GTSP) and focus on the case of the Lin-Kernighan algorithm. At first, we provide an easy-to-understand description of the original LinKernighan heuristic. Then we propose several adaptations, both trivial and complicated. Finally, we conduct a fair competition between all the variations of the Lin-Kernighan adaptation and some other GTSP heuristics.

It appears that our adaptation of the Lin-Kernighan algorithm for the GTSP reproduces the success of the original heuristic. Different variations of our adaptation outperform all other heuristics in a wide range of trade-offs between solution quality and running time, making Lin-Kernighan the state-of-the-art GTSP local search.
\end{abstract}

Keywords: Heuristics, Lin-Kernighan, Generalized Traveling Salesman Problem, Combinatorial Optimization.

\section{Introduction}

One of the most successful heuristic algorithms for the famous Traveling Salesman Problem (TSP) known so far is the Lin-Kernighan heuristic (Lin and Kernighan, 1973). It was proposed almost forty years ago but even nowadays it is the state-of-the-art TSP local search (Johnson and McGeoch, 2002).

In this paper we attempt to reproduce the success of the original TSP Lin-Kernighan heuristic for the Generalized Traveling Salesman Problem (GTSP), which is an important extension of TSP. In the TSP, we are given a set $V$ of $n$ vertices and weights $w(x \rightarrow y)$ of moving from a vertex $x \in V$ to a vertex $y \in V$. A feasible solution, or a tour, is a cycle visiting every vertex in $V$ exactly once. In the GTSP, we are given a set $V$ of $n$ vertices, weights $w(x \rightarrow y)$ of moving from $x \in V$ to $y \in V$ and a partition of $V$ into $m$

\footnotetext{
* Corresponding author

Email addresses: daniel.karapetyan@gmail.com(D. Karapetyan), gutin@cs.rhul.ac.uk (G. Gutin)
} 
nonempty clusters $C_{1}, C_{2}, \ldots, C_{m}$ such that $C_{i} \cap C_{j}=\varnothing$ for each $i \neq j$ and $\bigcup_{i} C_{i}=V$. A feasible solution, or a tour, is a cycle visiting exactly one vertex in every cluster. The objective of both TSP and GTSP is to find the shortest tour.

If the weight matrix is symmetric, i.e., $w(x \rightarrow y)=w(y \rightarrow x)$ for any $x, y \in V$, the problem is called symmetric. Otherwise it is an asymmetric GTSP. In what follows, the number of vertices in cluster $C_{i}$ is denoted as $\left|C_{i}\right|$, the size of the largest cluster is $s$, and Cluster $(x)$ is the cluster containing a vertex $x$. The weight function $w$ can be used for edges, paths $w\left(x_{1} \rightarrow x_{2} \rightarrow \ldots \rightarrow x_{k}\right)=w\left(x_{1} \rightarrow x_{2}\right)+w\left(x_{2} \rightarrow x_{3}\right)+\ldots+w\left(x_{k-1} \rightarrow\right.$ $x_{k}$ ), and cycles.

Since Lin-Kernighan is designed for the symmetric problem, we do not consider the asymmetric GTSP in this research. However, some of the algorithms proposed in this paper are naturally suited for both symmetric and asymmetric cases.

Observe that the TSP is a special case of the GTSP when $\left|C_{i}\right|=1$ for each $i$ and, hence, the GTSP is NP-hard. The GTSP has a host of applications in warehouse order picking with multiple stock locations, sequencing computer files, postal routing, airport selection and routing for courier planes and some others, see, e.g., (Fischetti et al., 1995, 1997; Laporte et al., 1996; Noon and Bean, 1991) and references therein.

A lot of attention was paid in the literature to solving the GTSP. Several researchers (Ben-Arieh et al., 2003; Laporte and Semet, 1999; Noon and Bean, 1993) proposed transformations of the GTSP into the TSP. At first glance, the idea to transform a little-studied problem into a well-known one seems to be natural; however, this approach has a very limited application. On the one hand, it requires exact solutions of the obtained TSP instances because even a near-optimal solution of such TSP may correspond to an infeasible GTSP solution. On the other hand, the produced TSP instances have quite an unusual structure which is difficult for the existing solvers. A more efficient way to solve the GTSP exactly is a branch-and-bound algorithm designed by Fischetti et al. (1997). This algorithm was able to solve instances with up to 89 clusters. Two approximation algorithms were proposed in the literature, but both of them are unsuitable for the general case of the problem, and the guarantied solution quality is unreasonably low for real-world applications, see (Bontoux et al., 2010) and references therein.

In order to obtain good (i.e., not necessarily exact) solutions for larger GTSP instances, one should use the heuristic approach. Several construction heuristics and local searches were discussed in (Bontoux et al,, 2010; Gutin and Karapetyan, 2010; Hu and Raidl, 2008; Renaud and Boctor, 1998; Snyder and Daskin, 2006) and some others. A number of metaheuristics were proposed by Bontoux et al. (2010); Gutin and Karapetyan (2010); Gutin et al. (2008); Huang et al. (2005); Pintea et al. (2007); Silberholz and Golden (2007); Snyder and Daskin (2006); Tasgetiren et al. (2007); Yang et al. (2008).

In this paper we thoroughly discuss possible adaptations of a TSP heuristic for the GTSP and focus on the Lin-Kernighan algorithm. The idea of the Lin-Kernighan algorithm was already successfully applied to the Multidimensional Assignment Problem (Balas and Saltzman, 1991; Karapetyan and Gutin, 2010). A straightforward adaptation for the GTSP was proposed by $\mathrm{Hu}$ and Raidl (2008); their algorithm constructs a set of TSP instances and solves all of them with the TSP Lin-Kernighan heuristic. Bontoux et al. (2010) apply the TSP Lin-Kernighan heuristic to the TSP tours induced by the GTSP tours. It will be shown in Section 3 that both of these approaches are relatively weak.

The Lin-Kernighan heuristic is a sophisticated algorithm adjusted specifically for the 
TSP. The explanation provided by Lin and Kernighan (1973) is full of details which complicate understanding of the main idea of the method. We start our paper from a clear explanation of a simplified TSP Lin-Kernighan heuristic (Section2) and then propose several adaptations of the heuristic for the GTSP (Section 3). In Section 4 we provide results of a thorough experimental evaluation of all the proposed Lin-Kernighan adaptations and discuss the success of our approach in comparison to other GTSP heuristics. In Section 5 we discuss the outcomes of the conducted research and select the state-of-the-art GTSP local searches.

\section{The TSP Lin-Kernighan Heuristic}

In this section we describe the TSP Lin-Kernighan heuristic $\left(\mathrm{LK}_{\mathrm{tsp}}\right)$. It is a simplified version of the original algorithm. Note that (Lin and Kernighan, 1973) was published almost 40 years ago, when modest computer resources, obviously, influenced the algorithm design, hiding the main idea behind the technical details. Also note that, back then, the 'goto' operator was widely used; this affects the original algorithm description. In contrast, our interpretation of the algorithm is easy to understand and implement.

$\mathrm{LK}_{\text {tsp }}$ is a generalization of the $k$-opt local search. The $k$-opt neighborhood $N_{k \text {-opt }}(T)$ includes all the TSP tours which can be obtained by removing $k$ edges from the original tour $T$ and adding $k$ different edges such that the resulting tour is feasible. Observe that exploring the whole $N_{k \text {-opt }}(T)$ takes $O\left(n^{k}\right)$ operations and, thus, with a few exceptions, only 2-opt and rarely 3-opt are used in practice (Johnson and McGeoch, 2002; Rego and Glover, 2006).

Similarly to $k$-opt, $\mathrm{LK}_{\mathrm{tsp}}$ tries to remove and insert edges in the tour but it explores only some parts of the $k$-opt neighborhood that deem to be the most promising. Consider removing an edge from a tour; this produces a path. Rearrange this path to minimize its weight. To close up the tour we only need to add one edge. Since we did not consider this edge during the path optimization, it is likely that its weight is neither minimized nor maximized. Hence, the weight of the whole tour is probably reduced together with the weight of the path. Here is a general scheme of $\mathrm{LK}_{\mathrm{tsp}}$ :

1. Let $T$ be the original tour.

2. For every edge $e \rightarrow b \in T$ do the following:

(a) Let $P=b \rightarrow \ldots \rightarrow e$ be the path obtained from $T$ by removing the edge $e \rightarrow b$.

(b) Rearrange $P$ to minimize its weight. Every time an improvement is found during this optimization, try to close up the path $P$. If it leads to a tour shorter than $T$, save this tour as $T$ and start the whole procedure again.

(c) If no tour improvement was found, continue to the next edge (Step 2).

In order to reduce the weight of the path, a local search is used as follows. On every move, it tries to break up the path into two parts, invert one of these parts, and then rejoin them (see Figure 11. In particular, the algorithm tries every edge $x \rightarrow y$ and selects the one which maximizes the gain $g=w(x \rightarrow y)-w(e \rightarrow x)$. If the maximum $g$ is positive, the corresponding move is an improvement and the local search is applied again to the improved path. 


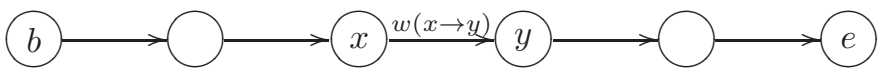

(a) The original path.

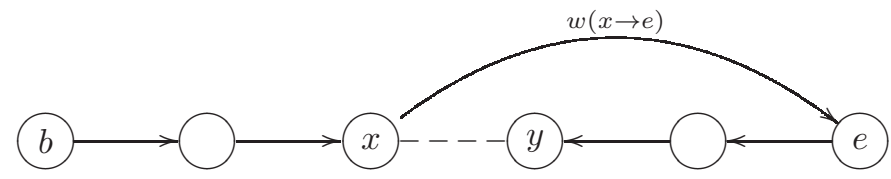

(b) The path after a local search move.

Figure 1: An example of a local search move for a path improvement. The weight of the path is reduced by $w(x \rightarrow y)-w(x \rightarrow e)$.

Observe that this algorithm tries only the best improvement and skips the other ones. A natural enhancement of the heuristic would be to use a backtracking mechanism to try all the improvements. However, this would slow down the algorithm too much. A compromise is to use the backtracking only for the first $\alpha$ moves. This approach is implemented in a recursive function ImprovePath $(P$, depth,$R)$, see Algorithm 1.

Algorithm 1 ImprovePath $(P$, depth,$R)$ recursive algorithm (LK tsp version). The function either terminates after an improved tour is found or finishes normally with no profit.

Require: The path $P=b \rightarrow \ldots \rightarrow e$, recursion depth depth and a set of restricted vertices $R$.

if depth $<\alpha$ then

for every edge $x \rightarrow y \in P$ such that $x \notin R$ do

Calculate $g=w(x \rightarrow y)-w(e \rightarrow x)$ (see Figure 1b).

if $g>0$ then

if the tour $b \rightarrow \ldots \rightarrow x \rightarrow e \rightarrow \ldots \rightarrow y \rightarrow b$ is an improvement over the original one then

Accept the produced tour and terminate.

else

else

ImprovePath $(b \rightarrow \ldots \rightarrow x \rightarrow e \rightarrow \ldots \rightarrow y$, depth $+1, R \cup\{x\})$.

Find the edge $x \rightarrow y$ which maximizes $g=w(x \rightarrow y)-w(e \rightarrow x)$.

if $g>0$ then

if the tour $b \rightarrow \ldots \rightarrow x \rightarrow e \rightarrow \ldots \rightarrow y \rightarrow b$ is an improvement over the original one then

Accept the produced tour and terminate.

else

return ImprovePath $(b \rightarrow \ldots \rightarrow x \rightarrow e \rightarrow \ldots \rightarrow y$, depth $+1, R \cup\{x\})$.

$\operatorname{ImprovePath}(P, 1, \varnothing)$ takes $O\left(n^{\alpha} \cdot\right.$ depth $\left._{\max }\right)$ operations, where depth $h_{\max }$ is the maximum depth of recursion achieved during the run. Hence, one should use only small values of backtracking depth $\alpha$. 
The algorithm presented above is a simplified Lin-Kernighan heuristic. Here is a list of major differences between the described algorithm and the original one.

1. The original heuristic does not accept the first found tour improvement. It records it and continues optimizing the path in the hope of finding a better tour improvement. Note that it was reported by Helsgaun (2000) that this complicates the algorithm but does not really improve its quality.

2. The original heuristic does not try all the $n$ options when optimizing a path. It considers only the five shortest edges $x \rightarrow e$ in the non-decreasing order. This hugely reduces the running time and helps to find the best rather than the first improvement on the backtracking stage. However, this speed-up approach is known to be a weak point of the original implementation (Helsgaun, 2000; Johnson and McGeoch, 2002). Indeed, even if the edge $x \rightarrow y$ is long, the algorithm does not try to break it if the edge $x \rightarrow e$ is not in the list of five shortest edges to $e$.

Note that looking for the closest vertices or clusters may be meaningless in the application to the GTSP. In our implementation, every edge $x \rightarrow y$ is considered.

3. The original heuristic does not allow deleting the previously added edges or adding the previously deleted edges. It was noted (Helsgaun, 2000; Johnson and McGeoch, 2002) that either of these restrictions is enough to prevent an infinite loop. In our implementation a previously deleted edge is allowed to be added again but every edge can be deleted only once. Our implementation also prevents some other moves; however, the experimental evaluation shows that this does not affect the performance of the heuristic.

4. The original heuristic also considers some more sophisticated moves to produce a path from the tour.

5. The original heuristic is, in fact, embedded into a metaheuristic which runs the optimization several times. There are several tricks related to the metaheuristic which are inapplicable to a single run.

The worst case time complexity of the Lin-Kernighan heuristic seems to be unknown from the literature (Helsgaun, 2009) but we assume that it is exponential. Indeed, observe that the number of iterations of the $k$-opt local search may be non-polynomial for any $k$ (Chandra et al., 1994) and that $\mathrm{LK}_{\mathrm{tsp}}$ is a modification of $k$-opt. However, Helsgaun (2009) notes that such undesirable instances are very rare and normally $L K_{\text {tsp }}$ proceeds in a polynomial time.

\section{Adaptations of the Lin-Kernighan Heuristic for the GTSP}

It may seem that the GTSP is only a slight variation of the TSP. In particular, one may propose splitting the GTSP into two problems (Renaud and Boctor, 1998): solving the TSP induced by the given tour to find the cluster order, and finding the shortest cycle visiting the clusters according to the found order. We will show now that this approach is poor with regards to solution quality. Let $N_{\mathrm{TSP}}(T)$ be a set of tours which can be obtained from the tour $T$ by reordering the vertices in $T$. Observe that one has to solve a TSP instance induced by $T$ to find the best tour in $N_{\mathrm{TSP}}(T)$.

Let $N_{\mathrm{CO}}(T)$ be a set of all the GTSP tours which visit the clusters in exactly the same order as in $T$. The size of the $N_{\mathrm{CO}}(T)$ neighborhood is $\prod_{i=1}^{m}\left|C_{i}\right| \in O\left(s^{m}\right)$ but there 
exists a polynomial algorithm (we call it Cluster Optimization, CO) which finds the best tour in $N_{\mathrm{CO}}(T)$ in $O\left(m s^{3}\right)$ operations (Fischetti et al., 1997). Moreover, it requires only $O\left(m s^{2} \cdot \min _{i}\left|C_{i}\right|\right)$ time, i.e., if the instance has at least one cluster of size $O(1)$, CO proceeds in $O\left(m s^{2}\right)$. (Recall that $s$ is the size of the largest cluster: $s=\max _{i}\left|C_{i}\right|$.)

The following theorem shows that splitting the GTSP into two problems (local search in $N_{\text {TSP }}(T)$ and then local search in $N_{\mathrm{CO}}(T)$ ) does not guarantee any solution quality.

Theorem 1. The best tour among $N_{C O}(T) \cup N_{T S P}(T)$ can be a longest GTSP tour different from a shortest one.

Proof. Consider the GTSP instance $G$ in Figure 2a, It is a symmetric GTSP containing 5 clusters $\{1\},\left\{2,2^{\prime}\right\},\{3\},\{4\}$ and $\{5\}$. The weights of the edges not displayed in the graph are as follows: $w(1 \rightarrow 3)=w(1 \rightarrow 4)=0$ and $w(2 \rightarrow 5)=w\left(2^{\prime} \rightarrow 5\right)=1$.

Observe that the tour $T=1 \rightarrow 2 \rightarrow 3 \rightarrow 4 \rightarrow 5 \rightarrow 1$, shown in Figure $2 \mathrm{~b}$ is a local minimum in both $N_{\mathrm{CO}}(T)$ and $N_{\mathrm{TSP}}(T)$. The dashed line shows the second solution in $N_{\mathrm{CO}}(T)$ but it gives the same objective value. It is also clear that $T$ is a local minimum in $N_{\text {TSP }}(T)$. Indeed, all the edges incident to the vertex 2 are of weight 1 , and, hence, any tour through the vertex 2 is at least of weight 2.

The tour $T$ is in fact a longest tour in $G$. Observe that all nonzero edges in $G$ are incident to the vertices 2 and $2^{\prime}$. Since only one of these vertices can be visited by a tour, at most two nonzero edges can be included into a tour. Hence, the weight of the worst tour in $G$ is 2 .

However, there exists a better GTSP tour $T_{\mathrm{opt}}=1 \rightarrow 2^{\prime} \rightarrow 4 \rightarrow 3 \rightarrow 5 \rightarrow 1$ of weight 1, see Figure 2a,

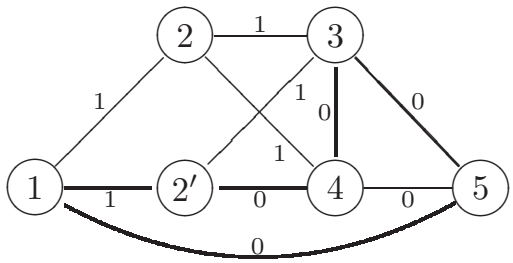

(a) The instance $G$ and the optimal GTSP tour $T_{\text {opt. }}$.

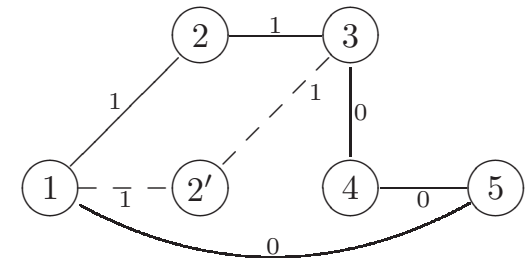

(b) A local minimum $T$ which is the worst possible GTSP tour.

Figure 2: An example of a local minimum in both $N_{\mathrm{TSP}}(T)$ and $N_{\mathrm{CO}}(T)$ which is a longest possible GTSP tour.

In fact, the TSP and the GTSP behave quite differently during optimization. Observe that there exists no way to find out quickly if some modification of the cluster order improves the tour. Indeed, choosing wrong vertices within clusters may lead to an arbitrary large increase of the tour weight. And since a replacement of a vertex within one cluster may require a replacement of vertices in the neighbor clusters, any local change influences the whole tour in general case.

\subsection{Local Search Adaptation}

A typical local search with the neighborhood $N(T)$ performs as follows: 
Require: The original solution $T$.

$$
\begin{aligned}
& \text { for all } T^{\prime} \in N(T) \text { do } \\
& \quad \text { if } w\left(T^{\prime}\right)<w(T) \text { then } \\
& \quad T \leftarrow T^{\prime} . \\
& \quad \text { Run the whole algorithm again. } \\
& \text { return } T \text {. }
\end{aligned}
$$

Let $N_{1}(T) \subseteq N_{\text {TSP }}(T)$ be a neighborhood of some TSP local search $L S_{1}(T)$. Let $N_{2}(T) \subseteq N_{\mathrm{CO}}(T)$ be a neighborhood of some GTSP local search $L S_{2}(T)$ which leaves the cluster order fixed. Then one can think of the following two adaptations of a TSP local search for the GTSP:

(i) Enumerate all solutions $T^{\prime} \in N_{1}(T)$. For every candidate $T^{\prime}$ run $T^{\prime} \leftarrow L S_{2}\left(T^{\prime}\right)$ to optimize it in $N_{2}\left(T^{\prime}\right)$.

(ii) Enumerate all solutions $T^{\prime} \in N_{2}(T)$. For every candidate $T^{\prime}$ run $T^{\prime} \leftarrow L S_{1}\left(T^{\prime}\right)$ to optimize it in $N_{1}\left(T^{\prime}\right)$.

Observe that the TSP neighborhood $N_{1}(T)$ is normally harder to explore than the cluster optimization neighborhood $N_{2}(T)$. Consider, e.g., $N_{1}(T)=N_{\text {TSP }}(T)$ and $N_{2}(T)=$ $N_{\mathrm{CO}}(T)$. Then both options yield an optimal GTSP solution but Option (il) requires $O\left(m ! m s^{3}\right)$ operations while Option (iii) requires $O\left(s^{m} m !\right)$ operations.

Moreover, many practical applications of the GTSP have some localization of clusters, i.e., $\left|w\left(x \rightarrow y_{1}\right)-w\left(x \rightarrow y_{2}\right)\right| \ll w\left(x \rightarrow y_{1}\right)$ on average, where Cluster $\left(y_{1}\right)=$ Cluster $\left(y_{2}\right) \neq \operatorname{Cluster}(x)$. Hence, the landscape of $N_{2}(T)$ depends on the cluster order more than the landscape of $N_{1}(T)$ depends on the vertex selection. From above it follows that Option (ii) is preferable.

Option (iii) was used by $\mathrm{Hu}$ and Raidl (2008) as follows. The cluster optimization neighborhood $N_{2}(T)$ includes there all the tours which differ from $T$ in exactly one vertex. For every $T^{\prime} \in N_{2}(T)$ the Lin-Kernighan heuristic was applied. This results in $n$ runs of the Lin-Kernighan heuristic which makes the algorithm unreasonably slow.

Option (ii) may be implemented as follows:

Require: The original tour $T$.

for all $T^{\prime} \in N_{1}(T)$ do

$T^{\prime} \leftarrow$ QuickImprove $\left(T^{\prime}\right)$.

if $w\left(T^{\prime}\right)<w(T)$ then

$T \leftarrow$ SlowImprove $\left(T^{\prime}\right)$.

Run the whole algorithm again.

return $T$.

Here QuickImprove $(T)$ and SlowImprove $(T)$ are some tour improvement heuristics which leave the cluster order unchanged. Formally, these heuristics should meet the following requirements:

- QuickImprove $(T)$, SlowImprove $(T) \in N_{\mathrm{CO}}(T)$ for any tour $T$;

- $w($ QuickImprove $(T)) \leq w(T)$ and $w(\operatorname{SlowImprove}(T)) \leq w(T)$ for any tour $T$.

QuickImprove is applied to every candidate $T^{\prime}$ before its evaluation. SlowImprove is only applied to successful candidates in order to further improve them. One can think of the following improvement functions: 
- Trivial $I(T)$ which leaves the solution without any change: $I(T)=T$.

- Full optimization $C O(T)$ which applies the $\mathrm{CO}$ algorithm to the given solution.

- Local optimization $L(T)$. It updates the vertices only within clusters, affected by the latest solution change. E.g., if a tour $x_{1} \rightarrow x_{2} \rightarrow x_{3} \rightarrow x_{4} \rightarrow x_{1}$ was changed to $x_{1} \rightarrow x_{3} \rightarrow x_{2} \rightarrow x_{4} \rightarrow x_{1}$, some implementation of $L(T)$ will try every $x_{1} \rightarrow x_{3}^{\prime} \rightarrow x_{2}^{\prime} \rightarrow x_{4} \rightarrow x_{1}$, where $x_{2}^{\prime} \in \operatorname{Cluster}\left(x_{2}\right)$ and $x_{3}^{\prime} \in \operatorname{Cluster}\left(x_{3}\right)$.

There are five meaningful combinations of QuickImprove and SlowImprove:

1. QuickImprove $(T)=I(T)$ and SlowImprove $(T)=I(T)$. This actually yields the original TSP local search.

2. QuickImprove $(T)=I(T)$ and SlowImprove $(T)=C O(T)$, i.e., the algorithm explores the TSP neighborhood but every time an improvement is found, the solution $T$ is optimized in $N_{\mathrm{CO}}(T)$. One can also consider SlowImprove $(T)=L(T)$, but it has no practical interest. Indeed, SlowImprove is used quite rarely and so its impact on the total running time is negligible. At the same time, $C O(T)$ is much better than $L(T)$ with respect to solution quality.

3. QuickImprove $(T)=L(T)$ and SlowImprove $(T)=I(T)$, i.e., every solution $T^{\prime} \in N(T)$ is improved locally before it is compared to the original solution.

4. QuickImprove $(T)=L(T)$ and SlowImprove $(T)=C O(T)$, which is the same as Option 3 but it additionally optimizes the solution $T^{\prime}$ globally in $N_{\mathrm{CO}}\left(T^{\prime}\right)$ every time an improvement is found.

5. QuickImprove $(T)=C O(T)$ and SlowImprove $(T)=I(T)$, i.e., every candidate $T^{\prime} \in N(T)$ is optimized globally in $N_{\mathrm{CO}}\left(T^{\prime}\right)$ before it is compared to the original solution $T$.

These adaptations were widely applied in the literature. For example, the heuristics G2 and G3 (Renaud and Boctor, 1998) are actually 2-opt and 3-opt adapted according to Option 5 An improvement over the naive implementation of 2-opt adapted in this way is proposed by $\mathrm{Hu}$ and Raidl (2008); asymptotically, it is faster by factor 3 . However, this approach is still too slow. Adaptations of 2-opt and some other heuristics according to Option 3 were used by Fischetti et al. (1997), Gutin and Karapetyan (2010), Silberholz and Golden (2007), Snyder and Daskin (2006), and Tasgetiren et al. (2007). Some unadapted TSP local searches (Option 11) were used by Bontoux et al. (2010), Gutin and Karapetyan (2010), Silberholz and Golden (2007), and Snyder and Daskin (2006).

\subsection{Adaptation of $L K_{t s p}$}

In this section we present our adaptation LK of $L K_{\text {tsp }}$ for the GTSP. A pseudo-code of the whole heuristic is presented in Algorithm 2 Some of its details are encapsulated into the following functions (note that $\mathrm{LK}_{\mathrm{tsp}}$ is not a typical local search based on some neighborhood and, thus, the framework presented above cannot be applied to it straightforwardly):

- $\operatorname{Gain}(P, x \rightarrow y)$ is intended to calculate the gain of breaking a path $P$ at an edge $x \rightarrow y$. 


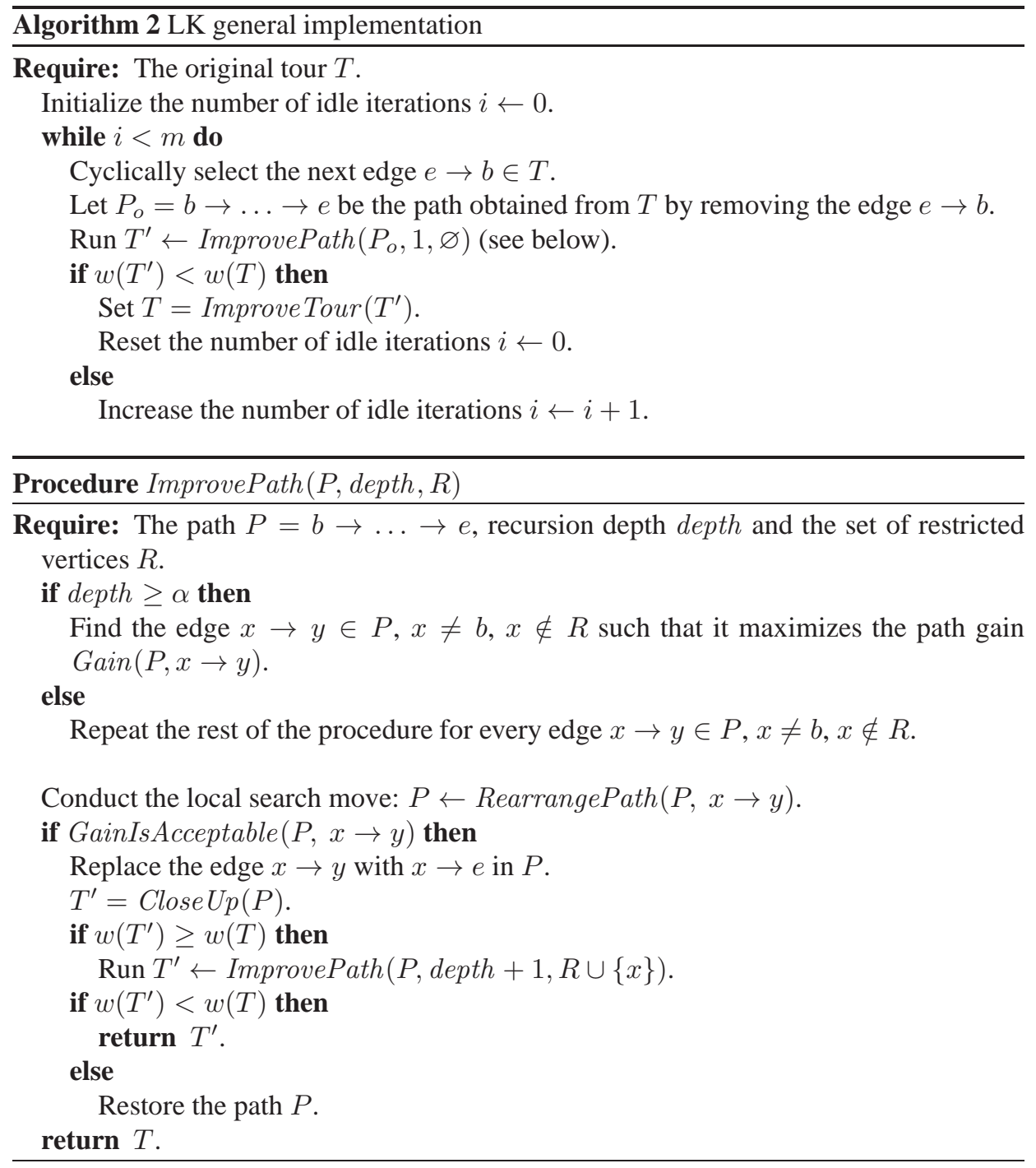

- RearrangePath $(P, x \rightarrow y)$ removes an edge $x \rightarrow y$ from a path $P$ and adds the edge $x \rightarrow e$, where $P=b \rightarrow \ldots \rightarrow x \rightarrow y \rightarrow \ldots \rightarrow e$, see Figure 1 . Together with CloseUp, it includes an implementation of QuickImprove $(T)$ (see Section 3.11, so RearrangePath may also apply some cluster optimization.

- GainIsAcceptable $(P, x \rightarrow y)$ determines if the gain of breaking a path $P$ at an edge $x \rightarrow y$ is worth any further effort.

- CloseUp $(P)$ adds an edge to a path $P$ to produce a feasible tour. Together with RearrangePath, it includes an implementation of QuickImprove(T) (see Section 3.1), so Close Up may also apply some cluster optimization. 
- ImproveTour $(T)$ is a tour improvement function. It is an analogue to SlowImprove $(T)$ (see Section 3.1).

These functions are the key points in the adaptation of $\mathrm{LK}_{\mathrm{tsp}}$ for the GTSP. They determine the behaviour of the heuristic. In Sections 3.3, 3.4 and 3.5 we describe different implementations of these functions.

\subsection{The Basic Variation}

The Basic variation of $\mathrm{LK}_{\mathrm{tsp}}$ (in what follows denoted by $\mathrm{B}$ ) is a trivial adaptation of LK according to Option 1 (see Section 3.1). It defines the functions Gain, RearrangePath, CloseUp and ImproveTour as follows:

$$
\begin{gathered}
\operatorname{Gain}_{\mathrm{B}}(b \rightarrow \ldots \rightarrow e, x \rightarrow y)=w(x \rightarrow y)-w(e \rightarrow x), \\
\text { RearrangePath }_{\mathrm{B}}(b \rightarrow \ldots \rightarrow x \rightarrow y \rightarrow \ldots \rightarrow e, x \rightarrow y)=b \rightarrow \ldots \rightarrow x \rightarrow e \rightarrow \ldots \rightarrow y, \\
\text { CloseUp }_{\mathrm{B}}(b \rightarrow \ldots \rightarrow e)=b \rightarrow \ldots \rightarrow e \rightarrow b,
\end{gathered}
$$

and $\operatorname{ImproveTour}_{\mathrm{B}}(T)$ is trivial. We also consider a $\mathrm{B}^{\mathrm{co}}$ variation (Option 2) which applies $\mathrm{CO}$ every time an improvement is found: $\operatorname{Improve} \operatorname{Tour}(T)=C O(T)$.

The implementation of GainIsAcceptable $(G, P)$ will be discussed in Section 3.6

\subsection{The Closest and the Shortest Variations}

The Closest and the Shortest variations (denoted as $\mathrm{C}$ and S, respectively) are two adaptations of $\mathrm{LK}_{\mathrm{tsp}}$ according to Option 3 i.e., QuickImprove $(T)=L(T)$ and SlowImprove $(T)=I(T)$. In other words, a local cluster optimization is applied to every candidate during the path optimization.

Consider an iteration of the path improvement heuristic ImprovePath. Let the path $P=b \rightarrow \ldots \rightarrow x \rightarrow y \rightarrow \ldots \rightarrow e$ be broken at the edge $x \rightarrow y$ (see Figure 3). Then,

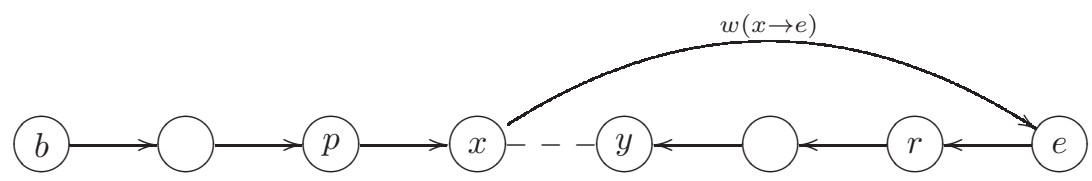

Figure 3: Path optimization.

to calculate $\operatorname{Gain}(P, x \rightarrow y)$ in $\mathrm{C}$, we replace $x \in X$ with $x^{\prime} \in X$ such that the edge $x \rightarrow e$ is minimized:

$$
\begin{aligned}
\operatorname{Gain}_{\mathrm{C}}(b \rightarrow \ldots \rightarrow p \rightarrow x \rightarrow y \rightarrow \ldots \rightarrow e, & x \rightarrow y) \\
& =w(p \rightarrow x \rightarrow y)-w\left(p \rightarrow x^{\prime} \rightarrow e\right),
\end{aligned}
$$

where $x^{\prime} \in \operatorname{Cluster}(x)$ is chosen to minimize $w\left(x^{\prime} \rightarrow e\right)$.

In $\mathrm{S}$, we update both $x$ and $e$ such that the path $p \rightarrow x \rightarrow e \rightarrow r$ is minimized:

$$
\begin{aligned}
& \operatorname{Gain}_{\mathrm{S}}(b \rightarrow \ldots \rightarrow p \rightarrow x \rightarrowy \rightarrow \ldots \rightarrow r \rightarrow e, x \rightarrow y)= \\
& w(p \rightarrow x \rightarrow y)+w(r \rightarrow e)-w\left(p \rightarrow x^{\prime} \rightarrow e^{\prime} \rightarrow r\right),
\end{aligned}
$$


where $x^{\prime} \in \operatorname{Cluster}(x)$ and $e^{\prime} \in$ Cluster $(e)$ are chosen to minimize $w\left(p \rightarrow x^{\prime} \rightarrow e^{\prime} \rightarrow\right.$ $r)$.

Observe that the most time-consuming part of LK is the path optimization. In case of the $\mathrm{S}$ variation, the bottleneck is the gain evaluation function which takes $O\left(s^{2}\right)$ operations. In order to reduce the number of gain evaluations in $\mathrm{S}$, we do not consider some edges $x \rightarrow y$. In particular, we assume that the improvement is usually not larger than $w_{\min }(X, Y)-w_{\min }(X, E)$, where $X=\operatorname{Cluster}(x), Y=\operatorname{Cluster}(y), E=\operatorname{Cluster}(e)$ and $w_{\min }(A, B)$ is the weight of the shortest edge between some clusters $A$ and $B$ : $w_{\min }(A, B)=\min _{a \in A, b \in B} w(a \rightarrow b)$. Obviously, all the values $w_{\min }(A, B)$ are precalculated. Note that this speed-up heuristic is used only when depth $\geq \alpha$, see Algorithm 2

One can hardly speed up the Gain function in B or C.

The RearrangePath function does some further cluster optimization in the $\mathrm{C}$ variation:

$$
\begin{aligned}
\text { RearrangePath }_{\mathrm{C}}(b \rightarrow \ldots \rightarrow p \rightarrow x \rightarrow y & \rightarrow \ldots \rightarrow e, x \rightarrow y) \\
& =b \rightarrow \ldots \rightarrow p \rightarrow x^{\prime} \rightarrow e \rightarrow \ldots \rightarrow y,
\end{aligned}
$$

where $x^{\prime} \in \operatorname{Cluster}(x)$ is chosen to minimize the weight $w\left(p \rightarrow x^{\prime} \rightarrow e\right)$. In $\mathbf{S}$ it just repeats the optimization performed for the Gain evaluation:

$$
\begin{aligned}
\text { RearrangePath }_{\mathrm{S}}(b \rightarrow \ldots \rightarrow p \rightarrow x & \rightarrow y \rightarrow \ldots \rightarrow r \rightarrow e, x \rightarrow y) \\
& =b \rightarrow \ldots \rightarrow p \rightarrow x^{\prime} \rightarrow e^{\prime} \rightarrow r \rightarrow \ldots \rightarrow y,
\end{aligned}
$$

where $x^{\prime} \in \operatorname{Cluster}(x)$ and $e^{\prime} \in$ Cluster $(e)$ are chosen to minimize $w\left(p \rightarrow x^{\prime} \rightarrow e^{\prime} \rightarrow\right.$ $r)$.

Every time we want to close up the path, both $\mathrm{C}$ and $\mathrm{S}$ try all the combinations of the end vertices to minimize the weight of the loop:

$$
\begin{aligned}
& \text { CloseUp }_{\mathrm{C}, \mathrm{S}}(b \rightarrow p \rightarrow \ldots \rightarrow q \rightarrow e)=b^{\prime} \rightarrow p \rightarrow \ldots \rightarrow q \rightarrow e^{\prime} \rightarrow b^{\prime}: \\
& b^{\prime} \in \text { Cluster }(b), e^{\prime} \in \text { Cluster }(e) \text { and } w\left(q \rightarrow e^{\prime} \rightarrow b^{\prime} \rightarrow p\right) \text { is minimized. }
\end{aligned}
$$

We also implemented the $\mathrm{C}^{\mathrm{co}}$ and $\mathrm{S}^{\mathrm{co}}$ variations such that $\mathrm{CO}$ is applied every time a tour improvement is found (see Option 4 above): $\operatorname{Improve} \operatorname{Tour}(T)=C O(T)$.

\subsection{The Exact Variation}

Finally we propose the Exact $(E)$ variation. For every cluster ordering under consideration it finds the shortest path from the first to the last cluster (via all clusters in that order). After closing up the path it always applies $\mathrm{CO}$ (see Option 5 above). However, it explores the neighborhood much faster than a naive implementation would do.

The Gain function for $\mathrm{E}$ is defined as follows:

$$
\begin{aligned}
& \operatorname{Gain}_{\mathrm{E}}(b \rightarrow \ldots \rightarrow x \rightarrow y \rightarrow \ldots \rightarrow e, x \rightarrow y)= \\
& w_{\mathrm{co}}(b \rightarrow \ldots \rightarrow x \rightarrow e \rightarrow \ldots \rightarrow y)-w_{\mathrm{co}}(b \rightarrow \ldots \rightarrow x \rightarrow y \rightarrow \ldots \rightarrow e),
\end{aligned}
$$


where $w_{\mathrm{co}}(P)$ is the weight of the shortest path through the corresponding clusters:

$$
w_{\mathrm{co}}\left(x_{1} \rightarrow x_{2} \rightarrow \ldots \rightarrow x_{m}\right)=\min _{x_{i}^{\prime} \in \operatorname{Cluster}\left(x_{i}\right), i=1, \ldots, m} w\left(x_{1}^{\prime} \rightarrow x_{2}^{\prime} \rightarrow \ldots \rightarrow x_{m}^{\prime}\right) .
$$

Note that ImprovePath runs this function sequentially for every $x \rightarrow y \in P$. In case of a naive implementation, it would take $O\left(\mathrm{~m}^{2} \mathrm{~s}^{2}\right)$ operations. Our implementation requires only $O\left(m s^{3}\right)$ operations but in practice it is much faster (almost $O\left(m s^{2}\right)$ ). Also note that typically $m \gg s$.

Our implementation proceeds as follows. Let $X_{1}, X_{2}, \ldots, X_{m}$ be the sequence of clusters in the given path (see Figure 4a). Let $l_{v}$ be the length of the shortest path from

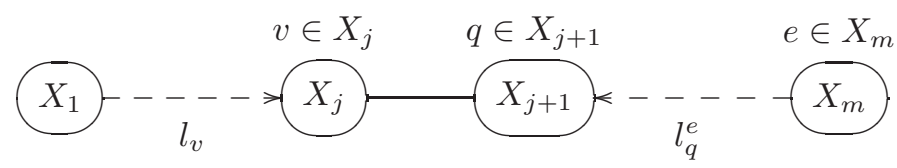

(a) The original sequence of clusters $X_{1}, X_{2}, \ldots, X_{m}$. The value $l_{v}$ denotes the shortest path from the cluster $X_{1}$ through $X_{2}, X_{3}, \ldots, X_{j-1}$ to the vertex $v \in X_{j}$. It takes $O\left(\left|X_{j-1}\right|\left|X_{j}\right|\right)$ operations to calculate all $l_{v}$ for some $j$. Value $l_{q}^{e}$ denotes the shortest path from the vertex $e \in X_{m}$ through $X_{m-1}, X_{m-2}, \ldots, X_{j+2}$ to the vertex $q \in X_{j+1}$. It takes $O\left(\left|X_{m}\right|\left|X_{j+2}\right|\left|X_{j+1}\right|\right)$ operations to calculate all $l_{q}^{e}$ for some $j$.

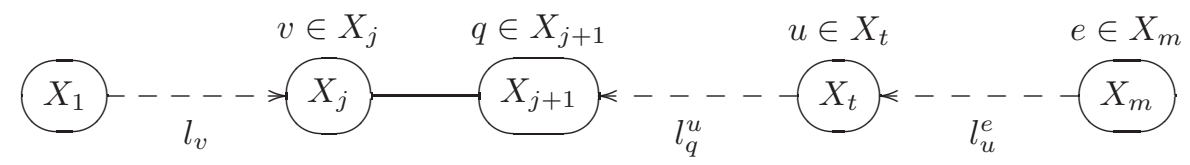

(b) An improved algorithm. Let cluster $X_{t}$ be the smallest cluster among $X_{j+2}, X_{j+3}, \ldots, X_{m}$. To calculate all the shortest paths $l_{q}^{u}$ from $u \in X_{t}$ to $q \in X_{j+1}$ via $X_{t-1}, X_{t-2}, \ldots, X_{j+2}$, one needs $O\left(\left|X_{t}\right|\left|X_{j+2}\right|\left|X_{j+1}\right|\right)$ operations for some $j$, i.e., it is $\left|X_{m}\right| /\left|X_{t}\right|$ times faster than the straightforward calculations. The values $l_{u}^{e}$ are calculated as previously, see Figure (a)

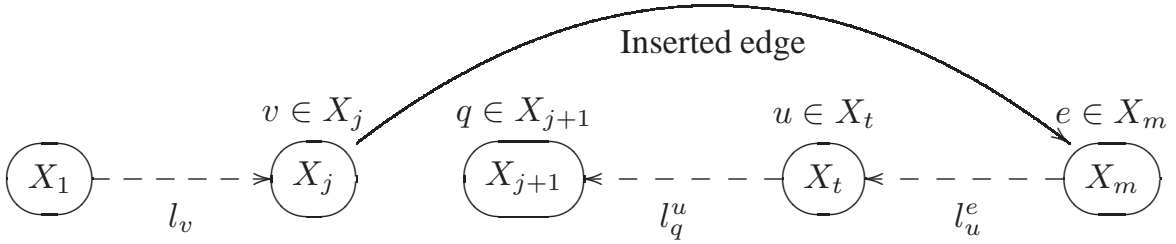

(c) The sequence of clusters after the local search move. To find the shortest path from $X_{1}$ to $X_{j+1}$ via $X_{2}, X_{3}, \ldots, X_{j}, X_{m}, X_{m-1}, \ldots, X_{j+2}$, we need to find all the shortest paths $l_{e}^{\prime}$ from $X_{1}$ to every $e \in X_{m}$ as $l_{e}^{\prime}=\min _{v}\left\{l_{v}+w(v \rightarrow e)\right\}$ in $O\left(s^{2}\right)$ operations, then find all the shortest paths $l_{u}^{\prime}$ from $X_{1}$ to every $u \in X_{t}$ as $l_{u}^{\prime}=\min _{e}\left\{l_{e}^{\prime}+l_{u}^{e}\right\}$ in $O\left(s^{2}\right)$ operations and, finally, find the whole shortest path $l^{\prime}$ from $X_{1}$ to $X_{j+1}$ as $l^{\prime}=\min _{u, q}\left\{l_{u}^{\prime}+l_{q}^{u}\right\}$ in $O\left(s^{2}\right)$ operations.

Figure 4: A straightforward and an enhanced implementations of the $E$ variation.

$X_{1}$ to $v \in X_{j}$ through the cluster sequence $X_{2}, X_{3}, \ldots, X_{j-1}$ :

$$
l_{v}=\min _{x_{i} \in X_{i}, i=1, \ldots, j-1} w\left(x_{1} \rightarrow x_{2} \rightarrow \ldots \rightarrow x_{j-1} \rightarrow v\right) .
$$


It takes $O\left(s^{2} m\right)$ operations to calculate all $l_{v}$ using the algorithm for the shortest path in layered networks.

Let $l_{q}^{e}$ be the length of the shortest path from $e \in X_{m}$ to $q \in X_{j+1}$ through the cluster sequence $X_{m-1}, X_{m-2}, \ldots, X_{j+2}$ :

$$
l_{q}^{e}=\min _{x_{i} \in X_{i}, i=j+2, \ldots, m-1} w\left(e \rightarrow x_{m-1} \rightarrow x_{m-2} \rightarrow \ldots \rightarrow x_{j+2} \rightarrow q\right) .
$$

It takes $O\left(s^{3} m\right)$ operations to calculate all $l_{q}^{e}$ using the algorithm for the shortest path in layered networks.

As a further improvement, we propose an algorithm to calculate $l_{q}^{e}$ which also takes $O\left(s^{3} m\right)$ operations in the worst case but in practice it proceeds significantly faster.

Note that a disadvantage of a straightforward use of the shortest path algorithm to find $l_{q}^{e}$ is that its performance strongly depends on the size of $X_{m}$; indeed, the straightforward approach requires $\left|X_{m}\right|\left|X_{j+2}\right|\left|X_{j+1}\right|$ operations for every $j$. Assume $\left|X_{t}\right|<\left|X_{m}\right|$ for some $t, j+1<t<m$, and we know the values $l_{u}^{e}$ for every $u \in X_{t}$ (see Figure 4b). Now for every $j<t-1$ we only need to calculate $l_{q}^{u}$, where $u \in X_{t}$ and $q \in X_{j+1}$. This will take $\left|X_{t}\right|\left|X_{j+1}\right|\left|X_{j}\right|$ operations for every $j$, i.e, it is $\left|X_{m}\right| /\left|X_{t}\right|$ times faster than the straightforward approach. A formal procedure is shown in Algorithm 3 .

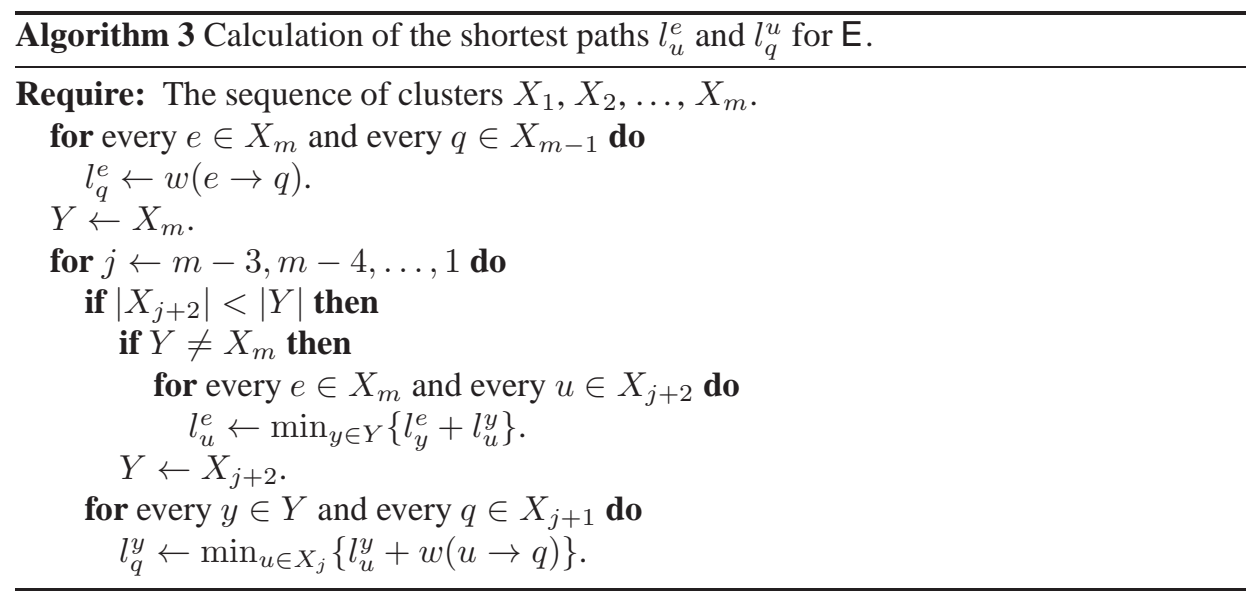

Having all $l_{v}, l_{u}^{e}$ and $l_{q}^{u}$, where $v \in X_{j}, q \in X_{j+1}, e \in X_{m}$ and $u \in X_{t}, j+1<t<$ $m$, one can find the shortest path through all the clusters $X_{1}, X_{2}, \ldots, X_{j}, X_{m}, X_{m-1}$, $\ldots, X_{j+1}$ in $O\left(s^{2}\right)$ time, see Algorithm 4 and Figure $4 \mathrm{c}$.

In our experiments this speed-up heuristic decreased the running time of the $E$ algorithm by $30 \%$ to $50 \%$.

The RearrangePath function for $\mathrm{E}$ replaces the edge $x \rightarrow y$ with $x \rightarrow e$ and optimizes the vertices in the path:

$$
\text { RearrangePath }_{\mathrm{E}}(b \rightarrow \ldots \rightarrow x \rightarrow y \rightarrow \ldots \rightarrow e)=b^{\prime} \rightarrow \ldots \rightarrow x^{\prime} \rightarrow y^{\prime} \rightarrow \ldots \rightarrow e^{\prime},
$$

where all the vertices are selected to minimize the weight of the resulting path. The Close $\mathrm{Up}$ function for $\mathrm{E}$ simply applies $\mathrm{CO}$ to the tour:

$$
\text { CloseUp } p_{\mathrm{E}}(b \rightarrow \ldots \rightarrow e)=C O(b \rightarrow \ldots \rightarrow e \rightarrow b) .
$$






Observe that, unlike other adaptations of the original $L K_{t s p}$ heuristic, Exact is naturally suitable for asymmetric instances.

Note that another approach to implement the $\mathrm{CO}$ algorithm is proposed by Pop (2007). It is based on an integer formulation of the GTSP; a more general case is studied in (Pop et al., 2006). However, we believe that the dynamic programming approach enhanced by the improvements discussed above is more efficient in our case.

\subsection{The Gain Function}

The gain is a measure of a path improvement. It is used to find the best path improvement and to decide whether this improvement should be accepted. To decide this, we use a boolean function $\operatorname{GainIsAcceptable}(P, x \rightarrow y)$. This function greatly influences the performance of the whole algorithm. We propose four different implementations of GainIsAcceptable $(P, x \rightarrow y)$ in order to find the most efficient ones. For the notation, see Algorithm 2

1. GainIsAcceptable $(P, x \rightarrow y)=w(P)<w\left(P_{o}\right)$, i.e., the function accepts any changes while the path is shorter than the original one.

2. GainIsAcceptable $(P, x \rightarrow y)=w(P)+\frac{w(T)}{m}<w(T)$, i.e., it is assumed that an edge of an average weight $\frac{w(T)}{m}$ will close up the path.

3. GainIsAcceptable $(P, x \rightarrow y)=w(P)+w(x \rightarrow y)<w(T)$, i.e., the last removed edge is 'restored' for the gain evaluation. Note that the weight of the edge $x \rightarrow y$ cannot be obtained correctly in $\mathrm{E}$. Instead of $w(x \rightarrow y)$ we use the weight $w_{\min }(X, Y)$ of the shortest edge between $X=$ Cluster $(x)$ and $Y=$ Cluster $(y)$.

4. GainIsAcceptable $(P, x \rightarrow y)=w(P)<w(T)$, i.e., the obtained path has to be shorter than the original tour. In other words, the weight of the 'close up edge' is assumed to be 0 . Unlike the first three implementations, this one is optimistic and, hence, yields deeper search trees. This takes more time but also improves the solution quality.

5. GainIsAcceptable $(P, x \rightarrow y)=w(P)+\frac{w(T)}{2 m}<w(T)$, i.e., it is assumed that an edge of a half of an average weight will close up the path. It is a mixture of Options 2 and 4 


\section{Experiments}

In order to select the most successful variations of the proposed heuristic and to prove its efficiency, we conducted a set of computational experiments.

Our test bed includes several TSP instances taken from TSPLIB (Reinelt, 1991) converted into the GTSP by the standard clustering procedure of Fischetti et al. (1997) (the same approach is widely used in the literature, see, e.g., (Gutin and Karapetyan, 2010; Silberholz and Golden, 2007; Snyder and Daskin, 2006; Tasgetiren et al., 2007)). Like Bontoux et al. (2010), Gutin and Karapetyan (2010), and Silberholz and Golden (2007), we do not consider any instances with less than 10 or more than 217 clusters (in other papers the bounds are stricter).

Every instance name consists of three parts: ' $m t n$ ', where $m$ is the number of clusters, $t$ is the type of the original TSP instance (see (Reinelt, 1991) for details) and $n$ is the number of vertices.

Observe that the optimal solutions are known only for some instances with up to 89 clusters (Fischetti et al., 1997). For the rest of the instances we use the best known solutions, see (Bontoux et al., 2010; Gutin and Karapetyan, 2010; Silberholz and Golden, 2007).

The following heuristics were included in the experiments:

1. The Basic variations, i.e., $\mathrm{B}_{x}^{\alpha}$ and $\mathrm{B}_{x}^{\alpha \text { co }}$, where $\alpha \in\{2,3,4\}$ and $x \in\{1,2,3,4,5\}$ define the backtracking depth and the gain acceptance strategy, respectively. The letters ' $\mathrm{co}$ ' in the superscript mean that the $\mathrm{CO}$ algorithm is applied every time a tour improvement is found (for details see Section 3.1).

2. The Closest variations, i.e., $\mathbf{C}_{x}^{\alpha}$ and $\mathbf{C}_{x}^{\alpha \text { co }}$, where $\alpha \in\{2,3,4\}$ and $x \in\{1,2,3,4,5\}$.

3. The Shortest variations, i.e., $\mathbf{S}_{x}^{\alpha}$ and $\mathbf{S}_{x}^{\alpha \text { co }}$, where $\alpha \in\{2,3,4\}$ and $x \in\{1,2,3,4,5\}$.

4. The Exact variations, i.e., $\mathbf{E}_{x}^{\alpha}$, where $\alpha \in\{1,2,3\}$ and $x \in\{1,2,3,4,5\}$.

5. Adaptations of the 2-opt (20) and 3-opt (30) local searches according to Section 3.1 .

6. A state-of-the-art memetic algorithm ma by Gutin and Karapetyan (2010).

Observe that ma dominates all other GTSP metaheuristics known from the literature. In particular, Gutin and Karapetyan (2010) compare it to the heuristics proposed by Silberholz and Golden (2007), Snyder and Daskin (2006) and Tasgetiren et al. (2007), and it appears that ma dominates all these algorithms in every experiment with respect to both solution quality and running time. Similarly, one can see that it dominates two more recent algorithms by Bontoux et al. (2010) and Tasgetiren et al. (2010) in every experiment. Note that the running times of all these algorithms were normalized according to the computational platforms used to evaluate the algorithms. Hence, we do not include the results of the other metaheuristics in our comparison.

In order to generate the starting tour for the local search procedures, we use a simplified Nearest Neighbour construction heuristic (NN). Unlike proposed by Noon (1988), our algorithm tries only one starting vertex. Trying every vertex as a starting one significantly slows down the heuristic and usually does not improve the solutions of the local searches. Note that in what follows the running time of a local search includes the running time of the construction heuristic. 
All the heuristics are implemented in Visual $\mathrm{C}++$. The evaluation platform is based on an Intel Core i7 $2.67 \mathrm{GHz}$ processor.

The experimental results are presented in two forms. The first form is a fair competition of all the heuristics joined in one table. The second form is a set of standard tables reporting solution quality and running time of the most successful heuristics.

\subsection{Heuristics Competition}

Many researchers face the problem of a fair comparison of several heuristics. Indeed, every experiment result consist of at least two parameters: solution error and running time. It is a trade-off between the speed and the quality, and both quick (and low-quality) and slow (and high-quality) heuristics are of interest. A heuristic should only be considered as useless if it is dominated by another heuristic, i.e., it is both slower and yields solutions of a lower quality.

Hence, one can clearly separate a set of successful from a set of dominated heuristics. However, this only works for a single experiment. If the experiment is conducted for several test instances, the comparison becomes not obvious. Indeed, a heuristic may be successful in one experiment and unsuccessful in another one. A natural solution of this problem is to use averages but if the results vary a lot for different instances this approach may be incorrect.

In a fair competition, one should compare heuristics which have similar running times. For every time $\tau_{i} \in\{0.02 \mathrm{~s}, 0.05 \mathrm{~s}, 0.1 \mathrm{~s}, 0.2 \mathrm{~s}, \ldots, 50 \mathrm{~s}\}$ we compare solution quality of all the heuristics which were able to solve an instance in less than $\tau_{i}$. In order to further reduce the size of the table and to smooth out the experimental results, we additionally group similar instances together and report only the average values for each group.

Moreover, we repeat every experiment 10 times. It requires some extra effort to ensure that an algorithm $H$ proceeds differently in different runs, i.e., $H^{i}(I) \neq H^{j}(I)$ in general case, where $i$ and $j$ are the run numbers. For $\mathbf{m a}^{r}$ the run number $r$ is the random generator seed value. In $\mathrm{NN}^{r}$, we start the tour construction from the vertex $C_{r, 1}$, i.e., from the first vertex of the $r$ th cluster of the instance. This also affects all the local searches since they start from the $\mathrm{NN}^{r}$ solutions.

Finally we get Table 1 Roughly speaking, every cell of this table reports the most successful heuristics for a given range of instances and being given some limited time. More formally, let $\tau=\left\{\tau_{1}, \tau_{2}, \ldots\right\}$ be a set of predefined time limits. Let $\mathcal{I}=\left\{\mathcal{I}_{1}, \mathcal{I}_{2}, \ldots\right\}$ be a set of predefined instance groups such that all instances in every $\mathcal{I}_{j}$ have similar difficulty. Let $\mathcal{H}$ be a set of all heuristics included in the competition. $H(I)_{\text {time }}$ and $H(I)_{\text {error }}$ are the running time and the relative solution error, respectively, of the heuristic $H \in \mathcal{H}$ for the instance $I \in \mathcal{I}$ :

$$
H(I)_{\mathrm{error}}=\frac{w(H(I))-w\left(I_{\text {best }}\right)}{w\left(I_{\text {best }}\right)},
$$

where $I_{\text {best }}$ is the optimal or the best known solution for the instance $I . H\left(\mathcal{I}_{j}\right)_{\text {time }}$ and $H\left(\mathcal{I}_{j}\right)_{\text {error }}$ denote the corresponding values averaged for all the instances $I \in \mathcal{I}_{j}$ and all $r \in\{1,2, \ldots, 10\}$.

For every cell $i, j$ we define a winner heuristic Winner $_{i, j} \in \mathcal{H}$ as follows:

1. Winner ${ }_{i, j}^{r}(I)_{\text {time }} \leq \tau_{i}$ for every instance $I \in \mathcal{I}_{j}$ and every $r \in\{1,2, \ldots, 10\}$. 
2. Winner $_{i, j}\left(\mathcal{I}_{j}\right)_{\text {error }}<$ Winner $_{i-1, j}\left(\mathcal{I}_{j}\right)_{\text {error }}$ (it is only applicable if $i>1$ ).

3. If several heuristics meet the conditions above, we choose the one with the smallest $H_{i, j}\left(\mathcal{I}_{j}\right)_{\text {error }}$.

4. If several heuristics meet the conditions above and have the same solution quality, we choose the one with the smallest $H_{i, j}\left(\mathcal{I}_{j}\right)_{\text {time }}$.

Apart from the winner, every cell contains all the heuristics $H \in \mathcal{H}$ meeting the following conditions:

1. $H^{r}(I)_{\text {time }} \leq \tau_{i}$ for every instance $I \in \mathcal{I}_{j}$ and every $r \in\{1,2, \ldots, 10\}$.

2. $H\left(\mathcal{I}_{j}\right)_{\text {error }}<$ Winner $_{i-1, j}\left(\mathcal{I}_{j}\right)_{\text {error }}$ (it is only applicable if $i>1$ ).

3. $H\left(\mathcal{I}_{j}\right)_{\text {error }} \leq 1.1 \cdot$ Winner $_{i, j}\left(\mathcal{I}_{j}\right)_{\text {error }}$.

4. $H\left(\mathcal{I}_{j}\right)_{\text {time }} \leq 1.2 \cdot$ Winner $_{i, j}\left(\mathcal{I}_{j}\right)_{\text {time }}$.

Since LK is a powerful heuristic, we did not consider any instances with less than 30 clusters in this competition. Note that all the smaller instances are relatively easy to solve, e.g., ma was able to solve all of them to optimality in our experiments, and it took only about $30 \mathrm{~ms}$ on average, and for $\mathrm{S}_{5}^{2 \mathrm{co}}$ it takes, on average, less than $0.5 \mathrm{~ms}$ to get $0.3 \%$ error, see Table 3

We use the following groups $\mathcal{I}_{j}$ of instances:

Tiniest: $30 \mathrm{ch} 150,30 \mathrm{kroA} 150,30 \mathrm{kroB} 150,31 \mathrm{pr} 152,32 \mathrm{u} 159$ and $39 \mathrm{rat} 195$. Tiny: $40 \mathrm{kroa} 200,40 \mathrm{krob} 200,41 \mathrm{gr} 202$, 45ts 225 , $45 \mathrm{tsp} 225$ and $46 \mathrm{pr} 226$. Small: 46gr229,53gil262, 56a280,60pr299 and 641 in 318.

Moderate: 80 rd400, 84 fl417, 87gr431, 88pr439 and 89pcb 442 .

Large: 99d493,107att532,107ali535,113pa561,115u574 and 115rat575. Huge: $132 \mathrm{~d} 657,134 \mathrm{gr} 666,145 \mathrm{u} 724$ and 157rat 783.

Giant: $200 d s j 1000,201$ pr1002,212u1060 and 217vm1084.

Note that the instances 35si175, 36brg180, $40 \mathrm{~d} 198$, 53pr264, 107si535, 131 p 654 and 207si1032 are excluded from this competition since they are significantly harder to solve than the other instances of the corresponding groups. This is discussed in Section 4.2 and the results for these instances are included in Tables 4 and 5.

One can see from Table 1 that there is a clear tendency: the proposed Lin-Kernighan adaptation outperforms all the other heuristics in a wide range of trade-offs between solution quality and running time. Only the state-of-the-art memetic algorithm ma is able to beat LK being given large time. There are several occurrences of 2-opt in the upper right corner (i.e., for Huge and Giant instances and less than $5 \mathrm{~ms}$ time) but this is because this time is too small for even the most basic variations of LK. Note that $2 \mathrm{o}_{\mathrm{B}}$ and $2 \mathrm{O}_{\mathrm{B}}^{\text {co }}$ denote the 2-opt local search adapted for the GTSP according to Options 1 and 2 respectively, see Section 3.1

Clearly, the most important parameter of LK is its variation, and each of the four variations (Basic, Closest, Shortest and Exact) is successful in a certain running time range. $\mathrm{B}$ wins the competition for small running times. For the middle range of running times one should choose $\mathrm{C}$ or $\mathrm{S}$. The $\mathrm{E}$ variation wins only in a small range of times; having more time, one should choose the memetic algorithm ma.

Here are some tendencies with regards to the rest of the LK parameters:

- It is usually beneficial to apply CO every time a tour improvement is found. 
Table 1: The fair competition. Every cell reports the most successful heuristics being given some limited time (see the first column) for a given range of instances (see the header). Every heuristic is provided with the average relative solution error in percent. To make the table easier to read, all the B and $E$ adaptations of LK are selected with bold font. All the cells where the dominating heuristic is $\mathrm{C}$ or $\mathrm{S}$ are highlighted with grey background.

\begin{tabular}{|c|c|c|c|c|c|c|c|}
\hline & Tiniest & Tiny & Small & Moderate & Large & Huge & Giant \\
\hline$\leq 2 \mathrm{~ms}$ & $\begin{array}{ll}\mathrm{S}_{4}^{2 \mathrm{co}} & 1.2 \\
\mathrm{~S}_{5}^{2 \mathrm{co}} & 1.2 \\
\mathrm{C}_{5}^{2 \mathrm{co}} & 1.3\end{array}$ & $\mathrm{C}_{5}^{2 \mathrm{co}} 1.0$ & $\mathrm{C}_{1}^{2 \mathrm{co}} 3.5$ & $\begin{array}{ll}\mathbf{B}_{5}^{2 \text { co }} & 6.1 \\
\mathbf{B}_{2}^{2 \text { co }} & 6.1 \\
\mathbf{B}_{1}^{400} & 6.3 \\
\mathbf{B}_{3}^{2 \text { co }} & 6.5\end{array}$ & $\mathrm{~B}_{1}^{2 \mathrm{co}} 7.8$ & $2 \mathrm{O}_{\mathrm{B}}^{\mathrm{co}} 13.4$ & $2 \mathrm{o}_{\mathrm{B}} 22.7$ \\
\hline$\leq 5 \mathrm{~ms}$ & $\mathrm{~S}_{5}^{3 \mathrm{co}} 0.0$ & $\mathrm{C}_{5}^{3 \mathrm{co}} 0.5$ & $\begin{array}{ll}\mathrm{C}_{5}^{3 \mathrm{co}} & 1.2 \\
\mathrm{~S}_{5}^{2 \mathrm{coo}} & 1.2\end{array}$ & $\mathrm{C}_{5}^{2 \mathrm{co}} 2.4$ & $\begin{array}{l}\mathbf{B}_{1}^{4 \text { co }} 7.2 \\
\mathbf{B}_{2}^{2 \text { co }} 7.3\end{array}$ & $\begin{array}{l}\mathbf{B}_{5}^{2 \text { co }} 9.5 \\
\mathbf{B}_{1}^{3 \operatorname{co}} 9.6 \\
\mathbf{B}_{\mathbf{3}}^{200} 10.1 \\
\mathbf{B}_{2}^{2 \text { co }} 10.3 \\
\end{array}$ & $2 \mathrm{o}_{\mathrm{B}}^{\mathrm{co}} 14.3$ \\
\hline$\leq 10 \mathrm{~ms}$ & - & - & $\mathrm{C}_{5}^{4 \mathrm{co}} 0.8$ & $\mathrm{C}_{4}^{2 \mathrm{co}} 1.3$ & $\mathrm{C}_{5}^{2 \mathrm{co}} 2.9$ & $\begin{array}{ll}\mathrm{C}_{1}^{2 \mathrm{co}} & 6.1 \\
\mathrm{C}_{2}^{2 \mathrm{co}} & 6.3\end{array}$ & $\mathbf{B}_{\mathbf{3}}^{2 \mathbf{c o}} 7.9$ \\
\hline$\leq 20 \mathrm{~ms}$ & - & $\mathrm{S}_{4}^{3 \mathrm{co}} 0.5$ & $\begin{array}{ll}\mathrm{S}_{5}^{3 \mathrm{co}} & 0.4 \\
\mathrm{~S}_{2}^{4 \mathrm{co}} & 0.5 \\
\end{array}$ & $\mathrm{C}_{5}^{3 \mathrm{co}} 1.3$ & $\mathrm{C}_{4}^{2 \mathrm{co}} 2.4$ & $\mathrm{C}_{5}^{2 \mathrm{co}} 4.0$ & - \\
\hline$\leq 50 \mathrm{~ms}$ & - & $\mathrm{S}_{4}^{4} \quad 0.2$ & $\mathrm{~S}_{5}^{4} \quad 0.2$ & $\mathrm{~S}_{2}^{4 \mathrm{co}} 1.1$ & $\begin{array}{ll}\mathrm{S}_{1}^{3} & 2.2 \\
\mathrm{~S}_{4}^{2 \mathrm{co}} & 2.2 \\
\end{array}$ & $\begin{array}{ll}\mathrm{S}_{5}^{2 \mathrm{co}} & 2.9 \\
\mathrm{C}_{5}^{3 \mathrm{co}} & 3.0 \\
\end{array}$ & $\mathrm{C}_{2}^{2 \mathrm{co}} 4.0$ \\
\hline$\leq 0.1 \mathrm{~s}$ & - & $\mathrm{S}_{4}^{4 \mathrm{co}} 0.2$ & $\mathrm{~S}_{4}^{4 \mathrm{co}} 0.0$ & - & $\mathrm{C}_{4}^{3 \mathrm{co}} 1.0$ & $\mathrm{C}_{4}^{3 \mathrm{co}} 1.7$ & $\mathrm{~S}_{2}^{2 \mathrm{co}} 3.0$ \\
\hline$\leq 0.2 \mathrm{~s}$ & - & - & - & $\begin{array}{ll}\mathbf{E}_{3}^{2} & 0.6 \\
\end{array}$ & - & - & $\mathrm{S}_{4}^{2 \mathrm{co}} 1.9$ \\
\hline$\leq 0.5 \mathrm{~s}$ & - & ma $\quad 0.0$ & - & - & - & $\mathrm{S}_{4}^{3 \mathrm{co}} 1.2$ & - \\
\hline$\leq 1 \mathrm{~s}$ & - & - & - & $\begin{array}{ll}\mathrm{E}_{5}^{3} & 0.4 \\
\end{array}$ & - & $\begin{array}{ll}\mathrm{E}_{2}^{3} & 1.0 \\
\end{array}$ & $\mathrm{~S}_{5}^{3 \mathrm{co}} 1.2$ \\
\hline$\leq 2 \mathrm{~s}$ & - & - & - & - & - & $\mathrm{S}_{4}^{4} \quad 1.0$ & - \\
\hline$\leq 5 \mathrm{~s}$ & - & - & - & $\begin{array}{ll}\mathrm{ma} & 0.0 \\
\end{array}$ & $\begin{array}{ll}\mathbf{E}_{\mathbf{5}}^{\mathbf{3}} & 0.8 \\
\end{array}$ & $\begin{array}{ll}\mathbf{E}_{\mathbf{3}}^{\mathbf{3}} & 0.8 \\
\end{array}$ & - \\
\hline$\leq 10 \mathrm{~s}$ & - & - & - & - & ma $\quad 0.0$ & - & - \\
\hline$\leq 20 \mathrm{~s}$ & - & - & - & - & - & ma $\quad 0.1$ & - \\
\hline$\leq 50 \mathrm{~s}$ & - & - & - & - & - & - & ma $\quad 0.2$ \\
\hline
\end{tabular}

- The most successful gain acceptance options are 4 and 5 (see Section 3.6).

- The larger the backtracking depth $\alpha$, the better the solutions. However, it is an expensive way to improve the solutions; one should normally keep $\alpha \in\{2,3,4\}$.

Table 1, however, does not make it clear what parameters one should use in practice. In order to give some advice, we calculated the distances $d(H)$ between each heuristic $H \in \mathcal{H}$ and the winner algorithms. For every column $j$ of Table 1 we calculated $d_{j}(H)$ :

$$
d_{j}(H)=\frac{H\left(\mathcal{I}_{j}\right)_{\text {error }}-\text { Winner }_{i, j}\left(\mathcal{I}_{j}\right)_{\text {error }}}{\text { Winner }_{i, j}\left(\mathcal{I}_{j}\right)_{\text {error }}},
$$

where $i$ is minimized such that $H^{r}(I)_{\text {time }} \leq \tau_{i}$ for every $I \in \mathcal{I}_{j}$ and $r \in\{1,2, \ldots, 10\}$. Then $d_{j}(H)$ were averaged for all $j$ to get the required distance: $d(H)=\overline{d_{j}(H)}$. The list 
of the heuristics $H$ with the smallest distances $d(H)$ is presented in Table 2 In fact, we added $2 \mathrm{O}_{\mathrm{B}}^{\mathrm{co}}, \mathrm{B}_{2}^{2 \mathrm{co}}$ and $\mathrm{E}_{4}^{2}$ to this list only to fill the gaps. Every heuristic $H$ in Table 2 is also provided with the average running time $T(H)$, in \% of ma running time:

$T(H)=\overline{T(H, I, r)}$ is averaged for all the instances $I \in \mathcal{I}$ and all $r \in\{1,2, \ldots, 10\}$, where $T(H, I, r)=\frac{H^{r}(I)_{\text {time }}}{M A(I)_{\text {time }}}$ and $M A(I)_{\text {time }}=\overline{M A^{r}(I)_{\text {time }}}$ is averaged for all $r \in\{1,2, \ldots, 10\}$.

Table 2: The list of the most successful heuristics. The heuristics $H$ are ordered according to their running times, from the fastest to the slowest ones. $2 \mathrm{O}_{\mathrm{B}}^{\text {co }}$ denotes the 2-opt local search adapted for the GTSP according to Option 2 see Section 3.1 .

\begin{tabular}{lrr}
\hline$H$ & $d(H), \%$ & Time, \% of ma time \\
\hline $2 \mathrm{O}_{\mathrm{B}}^{\text {co }}$ & 44 & 0.04 \\
$\mathrm{~B}_{2}^{2 \mathrm{co}}$ & 34 & 0.10 \\
$\mathrm{C}_{5}^{2 \mathrm{co}}$ & 12 & 0.40 \\
$\mathrm{~S}_{5}^{2 \mathrm{co}}$ & 19 & 0.97 \\
$\mathrm{~S}_{5}^{3 \text { co }}$ & 19 & 2.53 \\
$\mathrm{~S}_{5}^{4 \mathrm{co}}$ & 35 & 8.70 \\
$\mathrm{~S}_{4}^{4 \text { co }}$ & 32 & 15.34 \\
$\mathrm{E}_{4}^{2}$ & 56 & 43.62 \\
$\mathrm{ma}$ & 0 & 100.00 \\
\hline
\end{tabular}

\subsection{Detailed Data For Selected Heuristics}

In this section we provide the detailed information on the experimental results for the most successful heuristics, see Section 4.1. Tables 3,4 and 5 include the following information:

- The 'Instance' column contains the instance name as described above.

- The 'Best' column contains the best known or optimal (Fischetti et al., 1997) objective values of the test instances.

- The rest of the columns correspond to different heuristics and report either relative solution error or running time in milliseconds. Every value is averaged for ten runs, see Section 4.1 for details.

- The 'Average' row reports the averages for all the instances in the table.

- The 'Light avg' row reports the averages for all the instances used in Section 4.1

- Similarly, the 'Heavy avg' row reports the averages for all the instances ( $m \geq 30$ ) excluded from the competition in Section 4.1 . 
All the small instances $(m<30)$ are separated from the rest of the test bed to Table 3 One can see that all these instances are relatively easy to solve; in fact several heuristics are able to solve all or almost all of them to optimality in every run and it takes only a small fraction of a second. A useful observation is that $E_{4}^{2}$ solves all the instances with up to 20 clusters to optimality, and in this range $\mathrm{E}_{4}^{2}$ is significantly faster than ma.

As regards the larger instances ( $m \geq 30$ ), it is worth noting that there exist several 'heavy' instances among them: 35si175, 36 brg180, 40 d1 98, 53pr264, 107si 535, 131p654 and 207si1032. Some heuristics perform extremely slowly for these instances: the running time of $\mathrm{S}_{5}^{3 \mathrm{co}}, \mathrm{S}_{5}^{4 \mathrm{co}}, \mathrm{S}_{4}^{4 c o}$ and $\mathrm{E}_{4}^{2}$ is 3 to 500 times larger for every 'heavy" instance than it is for the other instances of a similar size. Other LK variations are also affected, though, this mostly relates to the ones which use the 'optimistic' gain acceptance functions (Options 4 and 5), see Section 3.6

Our analysis has shown that all of these instances have an unusual weight distribution. In particular, all these instances have enormous number of 'heavy' edges, i.e., the the weights which are close to the maximum weight in the instance, prevail over the smaller weights. Recall that LK bases on the assumption that a randomly selected edge will probably have a 'good' weight. Then we can optimize a path in the hope to find a good option to close it up later. However, the probability to find a 'good' edge is low in a 'heavy' instance. Hence, the termination condition GainIs Acceptable does not usually stop the search though a few tour improvements can be found. This, obviously, slows down the algorithm.

Note that a similar result was obtained by Karapetyan and Gutin (2010) for the adaptation of the Lin-Kernighan heuristic for the Multidimensional Assignment Problem.

Observe that such 'unfortunate' instances can be easily detected before the algorithm's run. Observe also that even the fast heuristics yield relatively good solutions for these instances (see Tables 4 and 5]. Hence, one can use a lighter heuristic to get a reasonable solution quality in a reasonable time in this case.

\section{Conclusion}

The Lin-Kernighan heuristic is known to be a very successful TSP heuristic. In this paper we present a number of adaptations of Lin-Kernighan for the GTSP. Several approaches to adaptation of a TSP local search for the GTSP are discussed and the best ones are selected and applied to the Lin-Kernighan heuristic. The experimental evaluation confirms the success of these approaches and proves that the proposed adaptations reproduce the efficiency of the original TSP heuristic.

Based on the experimental results, we selected the most successful Lin-Kernighan adaptations for different solution quality/running time requirements. Only for the very small running times ( $5 \mathrm{~ms}$ or less) and huge instances (132 clusters and more) our heuristic is outperformed by some very basic local searches just because none of our adaptations is able to proceed in this time. For the very large running times, the Lin-Kernighan adaptations are outperformed by the state-of-the-art memetic algorithm which usually solves the problem to optimality.

To implement the most powerful adaptation 'Exact', a new approach was proposed. Note that the same approach can be applied to many other TSP local searches. Comparing 
to the previous results in the literature, the time complexity of exploration of the corresponding neighborhood is significantly reduced which makes this adaptation practical. Though it was often outperformed by either faster adaptations or the memetic algorithm in our experiments, it is clearly the best heuristic for small instances (up to 20 clusters in our experiments) and it is also naturally suitable for the asymmetric GTSP.

Further research on adaptation of the Lin-Kernighan heuristic for other combinatorial optimization problems may be of interest. Our future plans also include a thorough study of different GTSP neighborhoods and their combinations.

\section{References}

Balas, E., Saltzman, M.J., 1991. An algorithm for the three-index assignment problem. Operations Research 39, 150-161.

Ben-Arieh, D., Gutin, G., Penn, M., Yeo, A., Zverovitch, A., 2003. Transformations of generalized ATSP into ATSP. Operations Research Letters 31, 357-365.

Bontoux, B., Artigues, C., Feillet, D., 2010. A memetic algorithm with a large neighborhood crossover operator for the generalized traveling salesman problem. Computers \& Operations Research 37, 1844-1852.

Chandra, B., Karloff, H., Tovey, C., 1994. New results on the old $k$-opt algorithm for the TSP, in: Proceedings of the 5th Annual ACM-SIAM Symposium on Discrete Algorithms, pp. 150-159.

Fischetti, M., Salazar González, J.J., Toth, P., 1995. The symmetric generalized traveling salesman polytope. Networks 26, 113-123.

Fischetti, M., Salazar González, J.J., Toth, P., 1997. A branch-and-cut algorithm for the symmetric generalized traveling salesman problem. Operations Research 45, 378-394.

Gutin, G., Karapetyan, D., 2010. A memetic algorithm for the generalized traveling salesman problem. Natural Computing 9, 47-60.

Gutin, G., Karapetyan, D., Krasnogor, N., 2008. A memetic algorithm for the generalized asymmetric traveling salesman problem, in: Nature Inspired Cooperative Strategies for Optimization (NICSO 2007), pp. 199-210.

Helsgaun, K., 2000. An effective implementation of the Lin-Kernighan traveling salesman heuristic. European Journal of Operational Research 126, 106-130.

Helsgaun, K., 2009. General k-opt submoves for the LinKernighan TSP heuristic. Mathematics and Statistics 1, 119-163.

Hu, B., Raidl, G.R., 2008. Effective neighborhood structures for the generalized traveling salesman problem, in: Proceedings of EvoCOP 2008, pp. 36-47.

Huang, H., Yang, X., Hao, Z., Wu, C., Liang, Y., Zhao, X., 2005. Hybrid chromosome genetic algorithm for generalized traveling salesman problems, in: Proceedings of ICNC 2005, pp. 137-140. 
Johnson, D.S., McGeoch, L.A., 2002. Experimental analysis of heuristics for the STSP, in: Gutin, G., Punnen, A.P. (Eds.), The Traveling Salesman Problem and its Variations. Kluwer, pp. 369-444.

Karapetyan, D., Gutin, G., 2010. Local search heuristics for the multidimensional assignment problem. To appear in Journal of Heuristics. A preliminary version is published in Lecture Notes Comp. Sci. 5420, pp. 100-115, 2009.

Laporte, G., Asef-Vaziri, A., Sriskandarajah, C., 1996. Some applications of the generalized travelling salesman problem. The Journal of the Operational Research Society 47, 1461-1467.

Laporte, G., Semet, F., 1999. Computational evaluation of a transformation procedure for the symmetric generalized traveling salesman problem. INFOR 37, 114-120.

Lin, S., Kernighan, B.W., 1973. An effective heuristic algorithm for the travelingsalesman problem. Operations Research 21, 498-516.

Noon, C.E., 1988. The Generalized Traveling Salesman Problem. Ph.D. thesis. University of Michigan.

Noon, C.E., Bean, J.C., 1991. A lagrangian based approach for the asymmetric generalized traveling salesman problem. Operations Research 39, 623-632.

Noon, C.E., Bean, J.C., 1993. An efficient transformation of the generalized traveling salesman problem. INFOR 31, 39-44.

Pintea, C., Pop, P., Chira, C., 2007. The generalized traveling salesman problem solved with ant algorithms. Journal of Universal Computer Science 13, 1065-1075.

Pop, P.C., 2007. New integer programming formulations of the generalized traveling salesman problem. American Journal of Applied Sciences 4, 932-937.

Pop, P.C., Kern, W., Still, G., 2006. A new relaxation method for the generalized minimum spanning tree problem. European Journal of Operational Research 170, 900-908.

Rego, C., Glover, F., 2006. Local search and metaheuristics, in: Gutin, G., Punnen, A. (Eds.), The Traveling Salesman Problem and Its Variations. Springer, pp. 309-368.

Reinelt, G., 1991. TSPLIB—a traveling salesman problem library. ORSA Journal on Computing 3, 376-384.

Renaud, J., Boctor, F.F., 1998. An efficient composite heuristic for the symmetric generalized traveling salesman problem. European Journal of Operational Research 108, $571-584$.

Silberholz, J., Golden, B.L., 2007. The generalized traveling salesman problem: A new genetic algorithm approach, in: Extending the Horizons: Advances in Computing, Optimization, and Decision Technologies. Springer, pp. 165-181. 
Snyder, L., Daskin, M., 2006. A random-key genetic algorithm for the generalized traveling salesman problem. European Journal of Operational Research 174, 38-53.

Tasgetiren, M., Suganthan, P.N., Pan, Q.Q., 2007. A discrete particle swarm optimization algorithm for the generalized traveling salesman problem, in: Proceedings of GECCO 2007, pp. 158-167.

Tasgetiren, M.F., Suganthan, P., Pan, Q.K., 2010. An ensemble of discrete differential evolution algorithms for solving the generalized traveling salesman problem. Applied Mathematics and Computation 215, 3356-3368.

Yang, J., Shi, X., Marchese, M., Liang, Y., 2008. An ant colony optimization method for generalized tsp problem. Progress in Natural Science 18, 1417-1422.

Table 3: Details of experiment results for the small (10 to 29 clusters) instances.

\begin{tabular}{|c|c|c|c|c|c|c|c|c|c|c|c|}
\hline \multirow[b]{2}{*}{ Instance } & \multirow[b]{2}{*}{ Best } & \multicolumn{5}{|c|}{ Solution error, $\%$} & \multicolumn{5}{|c|}{ Running time, ms } \\
\hline & & $2 \mathrm{o}_{\mathrm{B}}^{\mathrm{co}}$ & $\mathrm{C}_{5}^{2 \mathrm{co}}$ & $\mathrm{S}_{5}^{2 \mathrm{co}}$ & $\mathrm{E}_{4}^{2}$ & $\mathrm{ma}$ & $2 \mathrm{O}_{\mathrm{B}}^{\mathrm{co}}$ & $\mathrm{C}_{5}^{2 \mathrm{co}}$ & $\mathrm{S}_{5}^{2 \mathrm{co}}$ & $E_{4}^{2}$ & $\mathrm{ma}$ \\
\hline 10att48 & 5394 & 6.3 & 0.0 & 0.0 & 0.0 & 0.0 & 0.24 & 0.25 & 0.28 & 2.53 & 18.72 \\
\hline $10 \mathrm{gr} 48$ & 1834 & 4.9 & 0.0 & 0.0 & 0.0 & 0.0 & 0.01 & 0.03 & 0.06 & 1.45 & 12.48 \\
\hline 10hk48 & 6386 & 0.0 & 0.3 & 0.0 & 0.0 & 0.0 & 0.01 & 0.08 & 0.17 & 1.36 & 18.72 \\
\hline 11eil51 & 174 & 4.0 & 0.6 & 0.0 & 0.0 & 0.0 & 0.01 & 0.04 & 0.13 & 1.23 & 17.16 \\
\hline 11berlin52 & 4040 & 0.0 & 0.0 & 0.4 & 0.0 & 0.0 & 0.01 & 0.06 & 0.12 & 1.17 & 12.48 \\
\hline 12brazil58 & 15332 & 2.1 & 0.0 & 0.0 & 0.0 & 0.0 & 0.01 & 0.06 & 0.11 & 1.67 & 12.48 \\
\hline 14 st70 & 316 & 6.3 & 0.0 & 0.3 & 0.0 & 0.0 & 0.02 & 0.07 & 0.16 & 3.18 & 21.84 \\
\hline 16eil76 & 209 & 4.8 & 0.0 & 0.0 & 0.0 & 0.0 & 0.01 & 0.06 & 0.23 & 4.23 & 21.84 \\
\hline 16 pr76 & 64925 & 1.6 & 1.4 & 0.0 & 0.0 & 0.0 & 0.02 & 0.11 & 0.27 & 4.10 & 26.52 \\
\hline 20gr96 & 29440 & 2.4 & 1.0 & 0.0 & 0.0 & 0.0 & 0.03 & 0.22 & 0.42 & 9.09 & 28.08 \\
\hline 20rat99 & 497 & 7.8 & 0.2 & 0.0 & 0.0 & 0.0 & 0.03 & 0.24 & 0.64 & 15.05 & 37.44 \\
\hline 20kroa100 & 9711 & 4.2 & 5.8 & 0.0 & 0.0 & 0.0 & 0.03 & 0.17 & 0.46 & 14.59 & 31.20 \\
\hline 20krob100 & 10328 & 0.0 & 0.0 & 0.0 & 0.0 & 0.0 & 0.01 & 0.10 & 0.36 & 15.64 & 28.08 \\
\hline 20 kroc 100 & 9554 & 10.1 & 0.1 & 0.0 & 0.0 & 0.0 & 0.03 & 0.17 & 0.54 & 8.05 & 31.20 \\
\hline $20 \operatorname{krod} 100$ & 9450 & 1.5 & 0.0 & 2.0 & 0.0 & 0.0 & 0.05 & 0.17 & 0.44 & 10.12 & 39.00 \\
\hline 20kroe100 & 9523 & 1.3 & 4.4 & 0.0 & 0.0 & 0.0 & 0.03 & 0.15 & 0.37 & 8.33 & 31.20 \\
\hline $20 \mathrm{rd} 100$ & 3650 & 7.1 & 0.1 & 0.9 & 0.0 & 0.0 & 0.03 & 0.15 & 0.53 & 18.02 & 34.32 \\
\hline 21eil101 & 249 & 4.4 & 0.4 & 0.8 & 0.4 & 0.0 & 0.02 & 0.16 & 0.30 & 7.24 & 43.68 \\
\hline $21 \operatorname{lin} 105$ & 8213 & 0.1 & 0.0 & 0.0 & 0.0 & 0.0 & 0.02 & 0.14 & 0.36 & 5.50 & 32.76 \\
\hline 22pr107 & 27898 & 4.4 & 0.0 & 0.0 & 0.0 & 0.0 & 0.01 & 0.23 & 0.42 & 24.48 & 31.20 \\
\hline 24gr120 & 2769 & 20.5 & 2.8 & 2.6 & 0.0 & 0.0 & 0.03 & 0.24 & 0.77 & 10.77 & 43.68 \\
\hline $25 \mathrm{pr} 124$ & 36605 & 4.5 & 0.0 & 0.5 & 0.0 & 0.0 & 0.05 & 0.39 & 0.81 & 14.76 & 46.80 \\
\hline 26bier127 & 72418 & 6.9 & 8.6 & 0.0 & 0.0 & 0.0 & 0.08 & 0.36 & 0.69 & 12.45 & 54.60 \\
\hline $26 \operatorname{ch} 130$ & 2828 & 12.1 & 0.0 & 0.0 & 0.0 & 0.0 & 0.09 & 0.24 & 0.71 & 18.14 & 48.36 \\
\hline 28pr136 & 42570 & 9.7 & 0.8 & 0.0 & 0.0 & 0.0 & 0.04 & 0.49 & 0.77 & 14.24 & 49.92 \\
\hline $28 \operatorname{gr} 137$ & 36417 & 1.9 & 1.4 & 1.3 & 0.1 & 0.0 & 0.04 & 0.27 & 0.97 & 62.66 & 51.48 \\
\hline 29pr144 & 45886 & 4.0 & 0.0 & 0.0 & 0.0 & 0.0 & 0.03 & 0.36 & 0.58 & 15.31 & 40.56 \\
\hline Average & & 4.9 & 1.0 & 0.3 & 0.0 & 0.0 & 0.04 & 0.19 & 0.43 & 11.31 & 32.07 \\
\hline
\end{tabular}


Table 4: Detailed experiment results for the moderate and large instances $(m \geq 30)$. The reported values are relative solution errors, $\%$.

\begin{tabular}{|c|c|c|c|c|c|c|c|c|c|c|}
\hline Instance & Best & $2 \mathrm{O}_{\mathrm{B}}^{\mathrm{co}}$ & $\mathrm{B}_{2}^{2 \mathrm{co}}$ & $\mathrm{C}_{5}^{2 \mathrm{co}}$ & $\mathrm{S}_{5}^{2 \mathrm{co}}$ & $\mathrm{S}_{5}^{3 \mathrm{co}}$ & $\mathrm{S}_{5}^{4 \mathrm{co}}$ & $\mathrm{S}_{4}^{4 \mathrm{co}}$ & $E_{4}^{2}$ & $\mathrm{ma}$ \\
\hline $30 \operatorname{ch} 150$ & 2750 & 6.5 & 7.1 & 1.7 & 1.1 & 0.0 & 0.0 & 0.3 & 1.1 & 0.0 \\
\hline 30 kroa 150 & 11018 & 16.2 & 8.2 & 0.1 & 1.6 & 0.0 & 0.0 & 0.0 & 0.0 & 0.0 \\
\hline 30 krob 150 & 12196 & 5.4 & 5.4 & 0.0 & 1.0 & 0.0 & 0.5 & 0.0 & 0.0 & 0.0 \\
\hline 31 pr152 & 51576 & 4.1 & 3.3 & 3.9 & 1.9 & 0.0 & 0.0 & 0.0 & 1.2 & 0.0 \\
\hline $32 \mathrm{u} 159$ & 22664 & 24.9 & 10.2 & 0.8 & 0.4 & 0.0 & 0.0 & 0.0 & 1.1 & 0.0 \\
\hline $35 \operatorname{si} 175$ & 5564 & 2.6 & 3.8 & 0.0 & 0.0 & 0.0 & 0.0 & 0.0 & 0.0 & 0.0 \\
\hline 36 brg 180 & 4420 & 314.5 & 314.5 & 0.5 & 78.3 & 0.0 & 0.0 & 0.0 & 0.0 & 0.0 \\
\hline 39rat195 & 854 & 7.6 & 12.5 & 1.4 & 2.0 & 0.2 & 1.3 & 0.1 & 0.0 & 0.0 \\
\hline $40 \mathrm{~d} 198$ & 10557 & 1.3 & 3.5 & 1.3 & 0.3 & 0.0 & 0.5 & 0.0 & 0.2 & 0.0 \\
\hline $40 \mathrm{kroa} 200$ & 13406 & 8.3 & 4.8 & 0.6 & 0.4 & 0.4 & 0.4 & 0.4 & 0.0 & 0.0 \\
\hline 40 krob200 & 13111 & 14.6 & 14.0 & 0.1 & 2.7 & 0.2 & 0.1 & 0.0 & 0.0 & 0.0 \\
\hline 41 gr202 & 23301 & 10.5 & 7.1 & 3.1 & 4.3 & 2.5 & 1.9 & 0.0 & 0.0 & 0.0 \\
\hline $45 \mathrm{ts} 225$ & 68340 & 7.2 & 6.8 & 0.1 & 0.3 & 0.3 & 0.1 & 0.3 & 0.1 & 0.0 \\
\hline 45 tsp 225 & 1612 & 12.3 & 6.6 & 0.6 & 1.0 & 1.9 & 0.3 & 0.3 & 0.0 & 0.0 \\
\hline $46 \mathrm{pr} 226$ & 64007 & 14.2 & 1.1 & 1.1 & 1.1 & 0.0 & 0.0 & 0.0 & 0.0 & 0.0 \\
\hline $46 g r 229$ & 71972 & 7.6 & 8.1 & 1.2 & 1.0 & 0.0 & 0.0 & 0.0 & 0.9 & 0.0 \\
\hline 53 gil262 & 1013 & 20.6 & 11.1 & 3.6 & 0.7 & 0.8 & 0.7 & 0.0 & 0.2 & 0.0 \\
\hline 53 pr264 & 29549 & 9.9 & 0.7 & 0.8 & 0.8 & 1.0 & 0.4 & 0.2 & 0.5 & 0.0 \\
\hline $56 \mathrm{a} 280$ & 1079 & 5.9 & 3.3 & 2.3 & 0.8 & 0.3 & 0.3 & 0.0 & 0.6 & 0.0 \\
\hline 60pr299 & 22615 & 8.0 & 4.0 & 3.9 & 1.0 & 0.2 & 0.0 & 0.0 & 0.1 & 0.0 \\
\hline $64 \operatorname{lin} 318$ & 20765 & 10.1 & 8.3 & 3.7 & 2.5 & 0.9 & 0.0 & 0.0 & 2.6 & 0.0 \\
\hline $80 \mathrm{rd} 400$ & 6361 & 11.4 & 7.9 & 2.3 & 1.3 & 2.8 & 1.1 & 2.0 & 0.7 & 0.0 \\
\hline $84 f l 417$ & 9651 & 0.5 & 1.5 & 1.8 & 1.6 & 0.5 & 0.0 & 0.1 & 0.0 & 0.0 \\
\hline $87 \mathrm{gr} 431$ & 101946 & 5.1 & 5.2 & 2.6 & 3.2 & 3.6 & 2.5 & 1.1 & 0.0 & 0.0 \\
\hline $88 p r 439$ & 60099 & 9.7 & 5.9 & 1.8 & 1.2 & 1.4 & 1.3 & 0.0 & 1.1 & 0.0 \\
\hline 89pcb442 & 21657 & 7.8 & 5.5 & 2.9 & 0.1 & 1.0 & 0.0 & 1.7 & 2.1 & 0.0 \\
\hline $99 \mathrm{~d} 493$ & 20023 & 8.3 & 5.8 & 2.1 & 3.3 & 2.4 & 0.7 & 1.4 & 2.3 & 0.0 \\
\hline 107ali535 & 128639 & 15.9 & 5.0 & 3.1 & 2.6 & 0.5 & 0.0 & 0.4 & 0.5 & 0.0 \\
\hline 107att532 & 13464 & 11.3 & 5.6 & 0.8 & 1.5 & 0.5 & 0.8 & 0.1 & 0.1 & 0.0 \\
\hline $107 \mathrm{si} 535$ & 13502 & 2.4 & 1.3 & 0.3 & 0.1 & 0.1 & 0.0 & 0.0 & 0.3 & 0.0 \\
\hline 113pa561 & 1038 & 10.7 & 6.3 & 1.4 & 2.9 & 1.7 & 1.6 & 1.6 & 0.6 & 0.0 \\
\hline $115 u 574$ & 16689 & 10.4 & 9.5 & 5.7 & 5.1 & 0.2 & 1.1 & 1.0 & 1.6 & 0.0 \\
\hline 115 rat575 & 2388 & 13.4 & 11.5 & 4.4 & 4.2 & 3.5 & 3.2 & 3.2 & 1.3 & 0.2 \\
\hline 131 p654 & 27428 & 2.0 & 1.4 & 0.3 & 2.5 & 0.2 & 0.0 & 0.0 & 0.2 & 0.0 \\
\hline $132 \mathrm{~d} 657$ & 22498 & 10.6 & 9.5 & 4.6 & 3.9 & 1.7 & 1.6 & 0.5 & 1.9 & 0.1 \\
\hline 134gr666 & 163028 & 10.7 & 5.7 & 2.2 & 2.4 & 1.9 & 2.5 & 2.0 & 1.0 & 0.2 \\
\hline $145 \mathrm{u} 724$ & 17272 & 12.5 & 13.1 & 4.6 & 2.3 & 1.3 & 2.9 & 0.3 & 1.3 & 0.0 \\
\hline 157 rat783 & 3262 & 19.7 & 12.9 & 4.7 & 2.9 & 3.5 & 0.3 & 1.6 & 1.3 & 0.1 \\
\hline 200dsj1000 & 9187884 & 14.8 & 8.9 & 4.3 & 4.4 & 0.8 & 1.5 & 1.9 & 2.6 & 0.1 \\
\hline 201pr1002 & 114311 & 16.3 & 8.4 & 3.6 & 0.2 & 0.2 & 1.5 & 0.8 & 0.1 & 0.2 \\
\hline 207si1032 & 22306 & 5.2 & 4.1 & 1.7 & 1.2 & 0.9 & 0.1 & 0.1 & 0.9 & 0.0 \\
\hline $212 \mathrm{u} 1060$ & 106007 & 13.7 & 9.0 & 3.6 & 2.3 & 1.8 & 1.7 & 2.1 & 0.7 & 0.2 \\
\hline 217vm1084 & 130704 & 12.4 & 8.2 & 3.1 & 3.0 & 2.2 & 2.0 & 2.1 & 1.8 & 0.3 \\
\hline Average & & 17.1 & 13.9 & 2.2 & 3.6 & 1.0 & 0.8 & 0.6 & 0.7 & 0.0 \\
\hline Light avg. & & 11.4 & 7.6 & 2.5 & 2.0 & 1.1 & 0.9 & 0.7 & 0.8 & 0.0 \\
\hline Heavy avg. & & 42.3 & 41.3 & 0.8 & 10.6 & 0.3 & 0.1 & 0.1 & 0.3 & 0.0 \\
\hline
\end{tabular}


Table 5: Detailed experiment results for the moderate and large instances $(m \geq 30)$. The reported values are running times, ms.

\begin{tabular}{|c|c|c|c|c|c|c|c|c|c|}
\hline Instance & $2 \mathrm{o}_{\mathrm{B}}^{\mathrm{co}}$ & $\mathrm{B}_{2}^{2 \mathrm{co}}$ & $\mathrm{C}_{5}^{2 \mathrm{co}}$ & $\mathrm{S}_{5}^{2 \mathrm{co}}$ & $\mathrm{S}_{5}^{3 \mathrm{co}}$ & $\mathrm{S}_{5}^{4 \mathrm{co}}$ & $\mathrm{S}_{4}^{4 \mathrm{co}}$ & $\mathrm{E}_{4}^{2}$ & $\mathrm{ma}$ \\
\hline $30 \operatorname{ch} 150$ & 0.1 & 0.1 & 0.5 & 1.4 & 2.8 & 2.7 & 8.0 & 46.7 & 56.2 \\
\hline 30kroa150 & 0.0 & 0.1 & 0.4 & 1.0 & 1.8 & 4.0 & 4.2 & 32.3 & 57.7 \\
\hline 30 krob 150 & 0.0 & 0.1 & 0.4 & 1.2 & 1.5 & 2.5 & 7.0 & 50.6 & 65.5 \\
\hline 31 pr152 & 0.0 & 0.2 & 0.4 & 1.4 & 4.5 & 25.3 & 33.4 & 38.8 & 39.0 \\
\hline $32 \mathrm{u} 159$ & 0.1 & 0.1 & 0.3 & 0.9 & 2.7 & 4.2 & 25.7 & 31.9 & 62.4 \\
\hline $35 \operatorname{si} 175$ & 0.1 & 0.2 & 1.8 & 3.6 & 10.0 & 23.5 & 358.8 & 232.5 & 64.0 \\
\hline 36 brg 180 & 0.0 & 0.2 & 0.4 & 0.4 & 1.2 & 2.3 & 279.3 & 46.4 & 53.0 \\
\hline 39rat195 & 0.1 & 0.1 & 0.7 & 1.2 & 3.1 & 7.3 & 13.7 & 64.9 & 138.8 \\
\hline $40 \mathrm{~d} 198$ & 0.2 & 0.6 & 2.0 & 3.7 & 21.9 & 134.2 & 310.4 & 98.7 & 126.4 \\
\hline 40kroa200 & 0.1 & 0.1 & 0.7 & 1.6 & 3.2 & 4.1 & 11.7 & 60.6 & 123.2 \\
\hline 40 krob200 & 0.1 & 0.2 & 0.5 & 1.4 & 2.4 & 4.2 & 16.2 & 56.3 & 157.6 \\
\hline 41gr202 & 0.1 & 0.3 & 0.8 & 1.9 & 7.8 & 11.0 & 81.2 & 86.1 & 198.1 \\
\hline $45 \mathrm{ts} 225$ & 0.1 & 0.3 & 0.7 & 3.0 & 8.0 & 10.0 & 19.9 & 273.0 & 191.9 \\
\hline 45 tsp 225 & 0.1 & 0.2 & 0.8 & 2.3 & 3.1 & 7.6 & 15.5 & 112.3 & 156.0 \\
\hline $46 p r 226$ & 0.1 & 0.4 & 1.0 & 1.9 & 4.5 & 12.7 & 21.7 & 44.1 & 95.2 \\
\hline $46 g r 229$ & 0.1 & 0.2 & 1.0 & 3.2 & 3.5 & 8.8 & 13.9 & 145.1 & 224.6 \\
\hline 53 gil262 & 0.2 & 0.3 & 1.9 & 3.8 & 7.9 & 9.2 & 21.3 & 107.8 & 290.2 \\
\hline $53 p r 264$ & 0.2 & 1.0 & 5.7 & 6.5 & 66.2 & 282.4 & 505.4 & 230.9 & 204.4 \\
\hline $56 a 280$ & 0.2 & 0.3 & 1.1 & 2.2 & 11.2 & 9.3 & 43.9 & 148.2 & 291.7 \\
\hline 60pr299 & 0.1 & 0.2 & 1.5 & 3.8 & 8.7 & 12.6 & 31.4 & 146.7 & 347.9 \\
\hline $64 \operatorname{lin} 318$ & 0.2 & 0.3 & 2.0 & 4.2 & 17.3 & 48.6 & 81.4 & 223.1 & 404.0 \\
\hline $80 \mathrm{rd} 400$ & 0.3 & 0.7 & 3.8 & 5.6 & 18.2 & 36.7 & 74.4 & 305.8 & 872.0 \\
\hline $84 f l 417$ & 0.3 & 2.3 & 5.9 & 9.7 & 59.0 & 174.8 & 315.1 & 645.8 & 583.4 \\
\hline $87 \mathrm{gr} 431$ & 0.4 & 0.8 & 4.4 & 9.3 & 19.8 & 59.6 & 107.9 & 485.2 & 1673.9 \\
\hline 88pr439 & 0.3 & 0.8 & 3.0 & 11.6 & 24.4 & 54.3 & 109.3 & 764.4 & 1146.6 \\
\hline $89 p c b 442$ & 0.5 & 0.8 & 4.1 & 9.5 & 23.1 & 42.9 & 88.8 & 656.8 & 1530.4 \\
\hline $99 d 493$ & 0.7 & 2.0 & 7.5 & 13.1 & 148.3 & 2666.1 & 1616.2 & 591.2 & 3675.4 \\
\hline 107ali535 & 1.0 & 2.3 & 7.1 & 13.4 & 29.9 & 52.2 & 170.2 & 795.6 & 3558.4 \\
\hline 107att532 & 0.6 & 1.9 & 8.0 & 17.1 & 33.1 & 71.5 & 312.1 & 932.9 & 2942.2 \\
\hline $107 \mathrm{si} 535$ & 0.5 & 5.5 & 32.9 & 46.7 & 337.0 & 1921.9 & 12725.0 & 3503.8 & 1449.2 \\
\hline 113pa561 & 0.7 & 1.3 & 5.4 & 11.6 & 28.6 & 51.0 & 104.2 & 695.8 & 2931.3 \\
\hline $115 u 574$ & 0.7 & 1.9 & 6.8 & 10.7 & 53.3 & 63.9 & 156.1 & 956.3 & 3017.1 \\
\hline 115 rat575 & 0.5 & 1.3 & 6.4 & 17.9 & 41.0 & 92.9 & 128.0 & 697.3 & 2867.3 \\
\hline $131 \mathrm{p} 654$ & 1.2 & 9.4 & 40.9 & 27.3 & 213.9 & 1074.8 & 2964.0 & 3293.2 & 2137.2 \\
\hline $132 \mathrm{~d} 657$ & 0.9 & 2.3 & 13.6 & 22.0 & 109.4 & 1009.3 & 2322.9 & 794.0 & 4711.2 \\
\hline 134gr666 & 1.0 & 2.3 & 8.7 & 28.1 & 51.5 & 135.9 & 374.4 & 1425.8 & 10698.6 \\
\hline $145 \mathrm{u} 724$ & 1.0 & 2.7 & 13.4 & 32.6 & 62.8 & 105.8 & 242.0 & 1326.0 & 7952.9 \\
\hline 157 rat 783 & 1.5 & 2.2 & 17.7 & 30.8 & 73.7 & 131.3 & 248.3 & 2165.3 & 9459.9 \\
\hline 200dsj1000 & 3.5 & 10.3 & 80.7 & 104.5 & 592.8 & 5199.5 & 8032.5 & 9361.6 & 22704.4 \\
\hline 201pr1002 & 2.3 & 6.2 & 39.1 & 57.0 & 156.4 & 290.6 & 539.8 & 2719.1 & 21443.9 \\
\hline 207si1032 & 3.5 & 37.4 & 839.3 & 875.2 & 7063.7 & 195644.0 & 306944.8 & 112926.4 & 17840.3 \\
\hline $212 \mathrm{u} 1060$ & 3.7 & 7.1 & 36.4 & 80.2 & 195.5 & 307.5 & 1040.5 & 2990.5 & 31201.8 \\
\hline 217vm1084 & 2.5 & 6.6 & 51.4 & 78.5 & 204.8 & 496.1 & 978.1 & 4687.8 & 27587.2 \\
\hline Average & 0.7 & 2.6 & 29.3 & 36.3 & 226.4 & 4890.9 & 7941.8 & 3604.6 & 4310.1 \\
\hline Light avg. & 0.7 & 1.6 & 9.5 & 16.8 & 56.0 & 315.7 & 488.5 & 972.0 & 4653.6 \\
\hline Heavy avg. & 0.8 & 7.1 & 116.1 & 121.6 & 971.6 & 24907.3 & 40550.4 & 15122.2 & 2807.2 \\
\hline
\end{tabular}

